\title{
MARGINALLY STABLE INVISCID FLOWS WITH CRITICAL LAYERS*
}

\author{
K. STEWARTSON \\ Department of Mathematics, University College London, London, United Kingdom
}

\section{Introduction}

The inviscid theory of the stability of parallel shear flows is of immense importance to the understanding of a large class of fluid motions of scientific interest and has in consequence been the subject of very many papers during the last 100 years. It plays a central role in meteorology, oceanography and geophysics, to name a few of the more obvious applications. Naturally the linear theory has received most attention and it may be claimed that it is now in a fairly complete form, the student of any particular shear flow problem having at his disposal a sufficient number of theorems and special solutions to enable him to form a good understanding of the stability characteristics. An excellent review has been written some time ago by Drazin and Howard (1966) which provides an important background for this survey.

If the shear flow is unstable there is in general little that can be done beyond this stage, for non-linear terms assume importance on a relatively short time scale and the fluid motion might be expected to lose coherence quite soon with a band of unstable frequencies interacting and growing in a way which would defy, at the present time, even a numerical study.

The marginally stable flow appears to be more tractable in that the time scale of non-linear effects is long, due to the slow growth rate of any unstable linear disturbances. Hence it is possible to examine their importance compared with other effects such as initial conditions and viscosity. Further, one may study the possibility of the evolution of coherent structures or of catastrophic breakdown of the flow in fairly simple terms.

- Material used in this article first appeared in the IMA Journal of Applied Mathematics, Vol. 27 No 2 and is reproduced with permission. Copyright by Academic Press Inc. London Ltd. 


\section{Rayleigh's equation}

2.1. General comments. Let us begin by recalling some of the chief properties of linear stability theory. Define a Cartesian co-ordinate system $O x y z$ in which the fluid velocity is $\boldsymbol{u}^{*}$, the density $\varrho$ of the fluid is constant and the pressure is $p^{*}$. Then the governing equations are

$$
\frac{\partial u^{*}}{\partial t}+\left(u^{*} \nabla\right) u^{*}=-\frac{1}{\varrho} \nabla p^{*}, \quad \operatorname{div} u^{*}=0 .
$$

We suppose that the fluid is confined between two rigid planes $y=y_{1}, y_{2}$. either or both of which may be at infinity, and the basic flow is a shear in which $\boldsymbol{u}^{*}=\boldsymbol{u}_{B}^{*}$ where $\boldsymbol{u}_{B}^{*}$ has components $(U(y), 0,0)$ along the co-ordinate axes, and the pressure may be taken to be zero. Moreover, $U(y)$ is taken to be a smooth function of $y$, an assumption of crucial importance to the theory to be developed here, where the evolution of disturbances under non-linear and viscous forces is being considered. Thus piecewise continuous shears, which have been extensively studied during the last hundred years, are excluded from our discussion. In linear stability theory we make a small perturbation in the velocity so that $\boldsymbol{u}^{*}=\boldsymbol{u}_{B}^{*}+u$ where $u=(u, v, w)$, is sufficiently small that quadratic terms maybe neglected. Encouraged by Squire's theorem ( $\left.{ }^{1}\right)$ on the relation between two- and three-dimensional disturbances, we assume all dependent variables are independent of $z$, whence

$$
\begin{aligned}
\frac{\partial u}{\partial t}+U \cdot \frac{\partial u}{\partial x}+v \cdot \frac{\partial U}{\partial y} & =-\frac{1}{\varrho} \frac{\partial p^{*}}{\partial x}, \\
\frac{\partial v}{\partial t}+U \frac{\partial v}{\partial x} & =-\frac{1}{\varrho} \frac{\partial p^{*}}{\partial y}, \\
\frac{\partial u}{\partial x}+\frac{\partial v}{\partial y} & =0
\end{aligned}
$$

The corresponding boundary conditions for rigid walls are

$$
v=0 \quad \text { at } \quad y=y_{1}, y_{2} .
$$

For the stability problem we write

$$
u=\psi^{\prime}(y) e^{i x(x-c)}, \quad v=-i \alpha \psi(y) e^{i x(x-c r)}
$$

where $\alpha$ is real, $c$ has to be found, and

$$
(U-c)\left(\psi^{\prime \prime}-\alpha^{2} \psi\right)-U^{\prime \prime} \psi=0, \quad \psi\left(y_{1}\right)=\psi\left(y_{2}\right)=0 .
$$

This equation is known as Rayleigh's equation and the condition for

(1) Landhal (1980) has recently pointed out some special features of three-dimensional disturbances. 
instability of the basic flow to a disturbance of the form (2.4) is that $x c_{i}>0$ where $c=c_{r}+i c_{i}$.

Howard's semicircle theorem (1961) asserts that the possible values of the eigenvalues of this equation lie inside the circle

$$
\left[c_{r}-\frac{1}{2}\left(U_{\max }+U_{\min }\right)\right]^{2}+c_{i}^{2}=\left[\frac{1}{2}\left(U_{\max }-U_{\min }\right)\right]^{2},
$$

and, of course, the complex conjugate of $c$ is also an eigenvalue. If $c_{i}=0$ then the perturbation (2.4) is neutrally stable and Rayleigh's equation has a regular singularity in $y_{1}<y<y_{2}$ at $y=y_{c}$ where $U\left(y_{c}\right)=c_{r}$. There are now two alternatives. If $U^{\prime \prime}\left(y_{c}\right)=0$ then the singularity is removable and $\psi$ is a smooth function of $y$ near $y_{c}$. The mode (2.4) of oscillation is known as a regular neutral mode. On the other hand, if $U^{\prime \prime}\left(y_{c}\right) \neq 0$ the general solution for $\psi$ is of the form

$$
\psi_{c}+\frac{U^{\prime \prime}\left(y_{c}\right) \psi_{c}}{U^{\prime}\left(y_{c}\right)}\left(y-y_{c}\right)\left[\log \left|y-y_{c}\right|+i C_{ \pm}\right]+\ldots
$$

near $y=y_{c}$, where $C$, are constants appropriate to $y=y_{c}{ }^{5}$ and need not be equal. This mode is a singular neutral mode. The assumption of small disturbances now breaks down in the neighbourhood of $y=y_{c}$ and for this reason this point, at which the phase velocity of the disturbance is equal to the basic fluid velocity, is known as a critical level and its neighbourhood as the critical layer. The discontinuity $C_{+}-C_{-}$is usually referred to as the phase jump but we shall see that in certain important interpretations it is a function of $x$ and $t$, and so we shall describe it as a velocity jump. Since it implies a discontinuity, in the $x$-component of the perturbation velocity, across the critical level the new description has physical significance. In order to find an appropriate function $\psi$ when $c_{i}=0$, some assumption must be made about this velocity jump or the theory must be imbedded in a more comprehensive theory in which initial conditions, non-linear effects and viscosity are all taken into account.

To fix matters let us suppose that $U^{\prime \prime}(y) \leqslant 0$ in $y_{1}<y<y_{2}$. Then Rayleigh's criterion tells us that there are no solutions of $(2.5)$ with $c_{i} \neq 0$ and hence if there are any solutions they must have at least one critical level and possibly two. Further, the mode must be singular at one of these levels. There are three ways in which we can fix the conditions $\psi$ must satisfy across the critical layer, where linear theory, as presented so far, does not hold. One is to invoke both non-linear effects and viscosity in the critical layer. Benney and Bergeron (1969) have then shown that in certain circumstances which we shall discuss in detail later, we may set

$$
C_{+}=C_{-} \text {, }
$$

and now there is no difficulty about completing the solution of Rayleigh's equation for any $\alpha$. As an illustration consider the plane Poiseuille profile 
$U(y)=1-y^{2}$ with $y_{1}=-1, y_{2}=+1$ for which $U^{\prime \prime} \equiv-2$. The variation of $c_{r}$ with $\alpha$ for symmetric modes $(\psi(0)=0)$ is shown in Figure 1 and we see that $c_{r}=0$ when $\alpha=0$ and $c_{r} \rightarrow 1$ as $\alpha \rightarrow \infty$. Secondly, we may regard (2.5) as associated with the Laplace transform of the stream function which

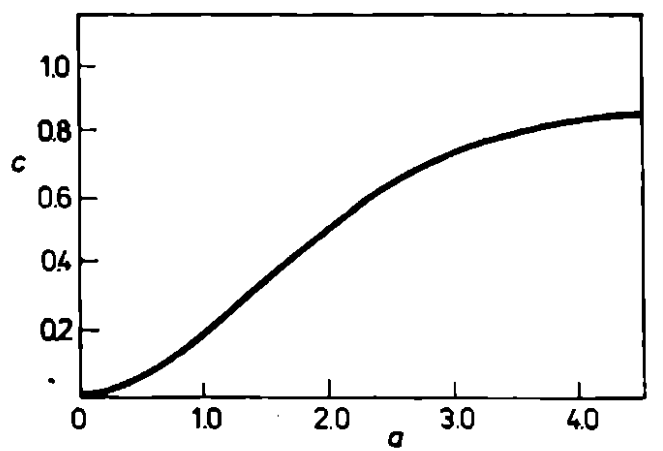

Fig. 1. Neutral curve for even disturbances for Poiseuille flow: $U(y)=1-y^{2},|y|<1 ; C_{+}=C_{-}$ (Benney and Bergeron (1969))

describes the evolution of the perturbation with increasing time after an initial perturbation proportional to exp(iax). Again we shall discuss this approach in more detail later but the matching condition across $y=y_{c}$ can be obtained by taking $c_{i}>0$ and letting $c_{i} \rightarrow 0$. It then follows that

$$
C_{+}=C_{-}+\pi \operatorname{sgn}\left(U^{\prime}\left(y_{c}\right)\right),
$$

or, if we define the Reynolds stress

$$
\tau=\frac{1}{4} i \alpha\left(\psi \psi^{\prime}-\psi^{\prime} \bar{\psi}\right)-\overline{u v},
$$

the tilde denoting the complex conjugate, the jump in $\tau$ across the critical layer is

$$
[\tau]_{-}^{+}=\frac{\alpha \pi}{2} \frac{U^{\prime \prime}\left(y_{c}\right)\left|\psi_{c}\right|^{2}}{\left|U^{\prime}\left(y_{c}\right)\right|} .
$$

Now $d \tau / d y=0$ elsewhere in $y_{1}<y<y_{2}$ and $\tau$ must vanish on the boundaries. A contradiction follows whether there is one critical level or two (the maximum), since the right-hand side of (2.11) is negative. We conclude that there are no solutions of (2.5) regarded as the limit of an initial value problems if $U^{\prime \prime}(y)$ does not change sign. The third possibility is to regard $\psi$ as a function of a complex variable $y$ which is then analytic in the finite $y$-plane except for a branch point at $y_{c}$ which may now be complex. We determine the eigenvalue $c$ by taking a path from $y_{1}$ to $y_{2}$ in the complex plane of $y$ passing below $y_{c}$. Having found $c$ by this means we then appeal to Cauchy's theorem to conclude that the same result would hold if the contour were deformed back to the real axis of $y$, provided that $y_{c}>0$. This method works well for unstable modes of inflexional profiles (Gill and Davey (1969); 
see also Lessen, Singh and Paillet (1974)), but for monotonic stable shear flows it leads to values of $c$ which contradict the requirement $\operatorname{Im} y_{c}>0$. No doubt if the contour were taken above $y_{c}$ we should then find that $\operatorname{Im} y_{c}>0$. Thus the solution of the Rayleigh equation pertinent to these stable flows must always have a cut at some point on the real axis across which $\psi$ and/or $\partial \psi / \partial y$ is discontinuous. It appears therefore that these solutions are quite irrelevant to the inviscid stability problem $\left({ }^{1}\right)$ but we shall now show that the value of $c$ obtained by taking the contour of integration below the real axis of $y$ is the limit of an eigenvalue of the Orr-Sommerfeld equation for viscous flow as is the eigensolution except for a finite stretch of the real $y$-axis.

2.2. Viscous effects in shear flows. In general, the inclusion of viscous effects in shear flow studies leads to an immediate contradiction with the assumption of parallel flow for the basic velocity profile does not satisfy the Navier-Stokes equations of motion. Notable among the few exceptions are Poiseuille flow, in which $U=1-y^{2},|y|<1$, Couette flow in which $U=y$, and Ekman boundary layers (Lilly (1966)) of which the last example is outside the scope of this review since the direction of the fluid velocity varies with $y$. The common practice, which we follow, is to ignore this contradiction and then after making assumptions equivalent to (2.4), we find that $\psi$ satisfies

$$
\left(\psi^{\prime \prime \prime \prime}-2 \alpha^{2} \psi^{\prime \prime}+\alpha^{4} \psi\right)-i \alpha R\left[(U-c)\left(\psi^{\prime \prime}-\alpha^{2} \psi\right)-U^{\prime \prime} \psi\right]=0
$$

where $R$ is a representative Reynolds number of the flow and

$$
\psi\left(y_{1}\right)=\psi^{\prime}\left(y_{1}\right)=\psi\left(y_{2}\right)=\psi^{\prime}\left(y_{2}\right)=0 .
$$

This equation is the famous Orr-Sommerfeld equation of fluid dynamical instability and we see that formally Rayleigh's equation is obtained in the limit $R \rightarrow \infty$. Some brief remarks on the general properties of solutions of (2.12) are in order before we discuss their limiting behaviour as $R \rightarrow \infty$. If the range $y_{1}<y<y_{2}$ of the solution is finite, there are an infinite number of solutions of (2.12) at any Reynolds number and the evolution of any initial disturbance is representable by a combination of these eigensolutions. On the other hand, if the range is infinite there is no guarantee that at a particular value of $R$ there are any solutions of (2.12) at all (Murdock and Stewartson (1977)). It seems that the central role in describing the evolution of an initial disturbance is provided by the continuous spectrum in which $\psi$ oscillates finitely as $y \rightarrow \infty$ and corresponds to a set of eigenfunctions obtained by a different method of separating variables (assuming that the dependent

(1) Some writers (e.g, Landahl (1980)) think otherwise but such discontinuities violate the principle conservation of mass and/or momentum. It has not proved possible to smear them out with solutions drawn from the continuous spectrum (see Section 2.4, below). 
variables are products of functions of $x$, of $t$, and of $y / \sqrt{t}$ instead of functions of $x, t$ and of $y$ ). In addition, as $R$ increases, a set of solutions of (2.12) springing off from this continuous spectrum at discrete values of $R$ is obtained. The phenomenon is clearly shown in Figure 2, taken from Mack's (1976) extensive computations at $\alpha=0,179$ for Blasius flow.
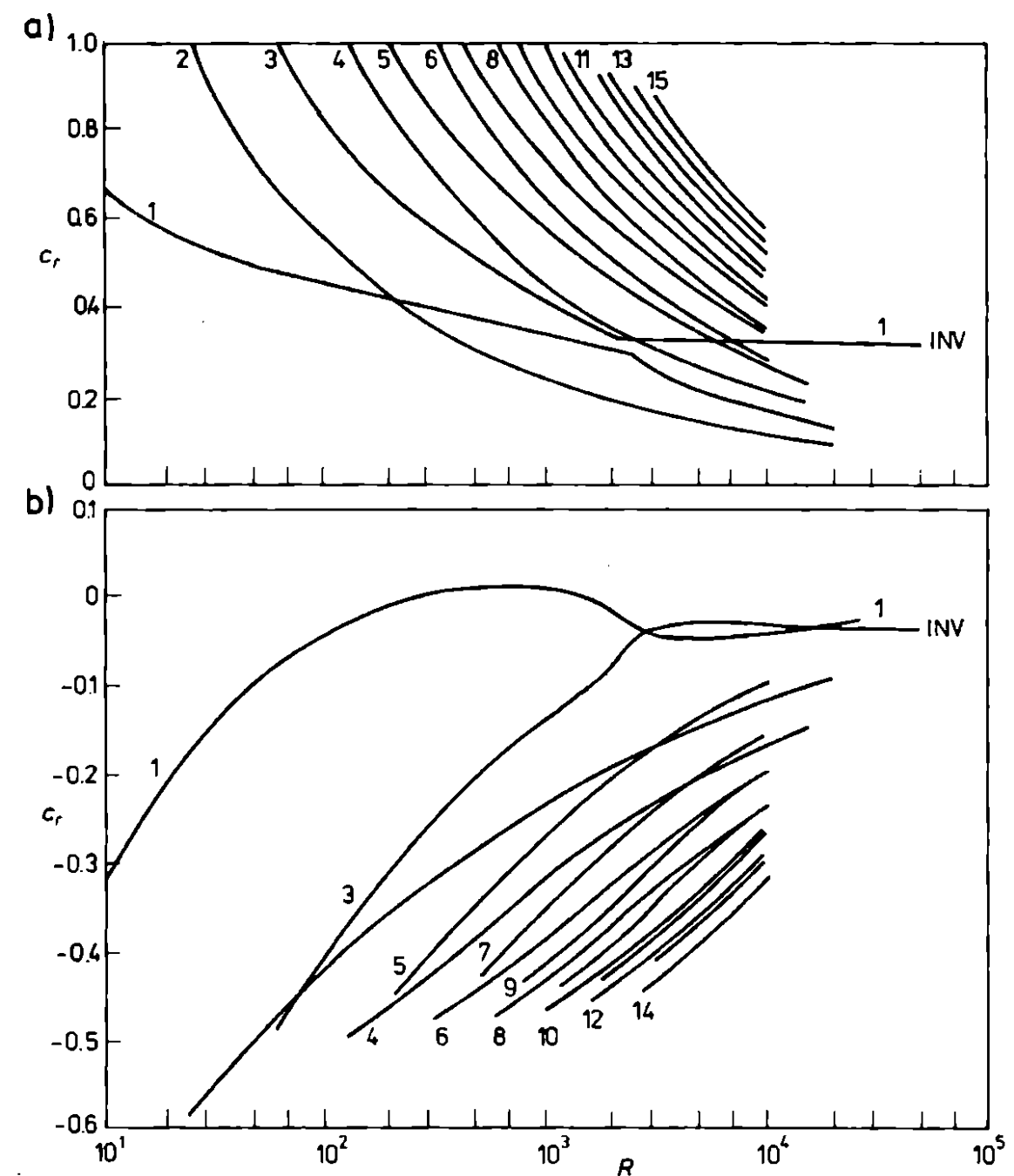

Fig. 2. Effect of Reynolds number on $c_{r}$ and $c_{i}$ of the first 15 modes of Blasius flow at $\alpha=0.179$, INV denotes eigenvalues according to inviscid theory but deforming the contour (Mack (1976))

The study of the limiting form of a solution of (2.12) as $R \rightarrow x$ has been carried out chiefly in two cases. For Poiseuille flow Grosch and Salwen (1968) have carried out a study of a considerable number of the eigenvalues at $\alpha=1$ up to values of $R \doteq 10000$ and found that $c$ is either approaching zero or unity (Fig. 3). Davey (1973) has extended these calculations for one particular mode in plane Poiseuille flow and has verified the asymptotic formula

$$
c \sim(6.56-1.68 i)\left(\frac{1}{R}\right)^{1 / 3} \quad \text { as } \quad R \rightarrow \infty
$$



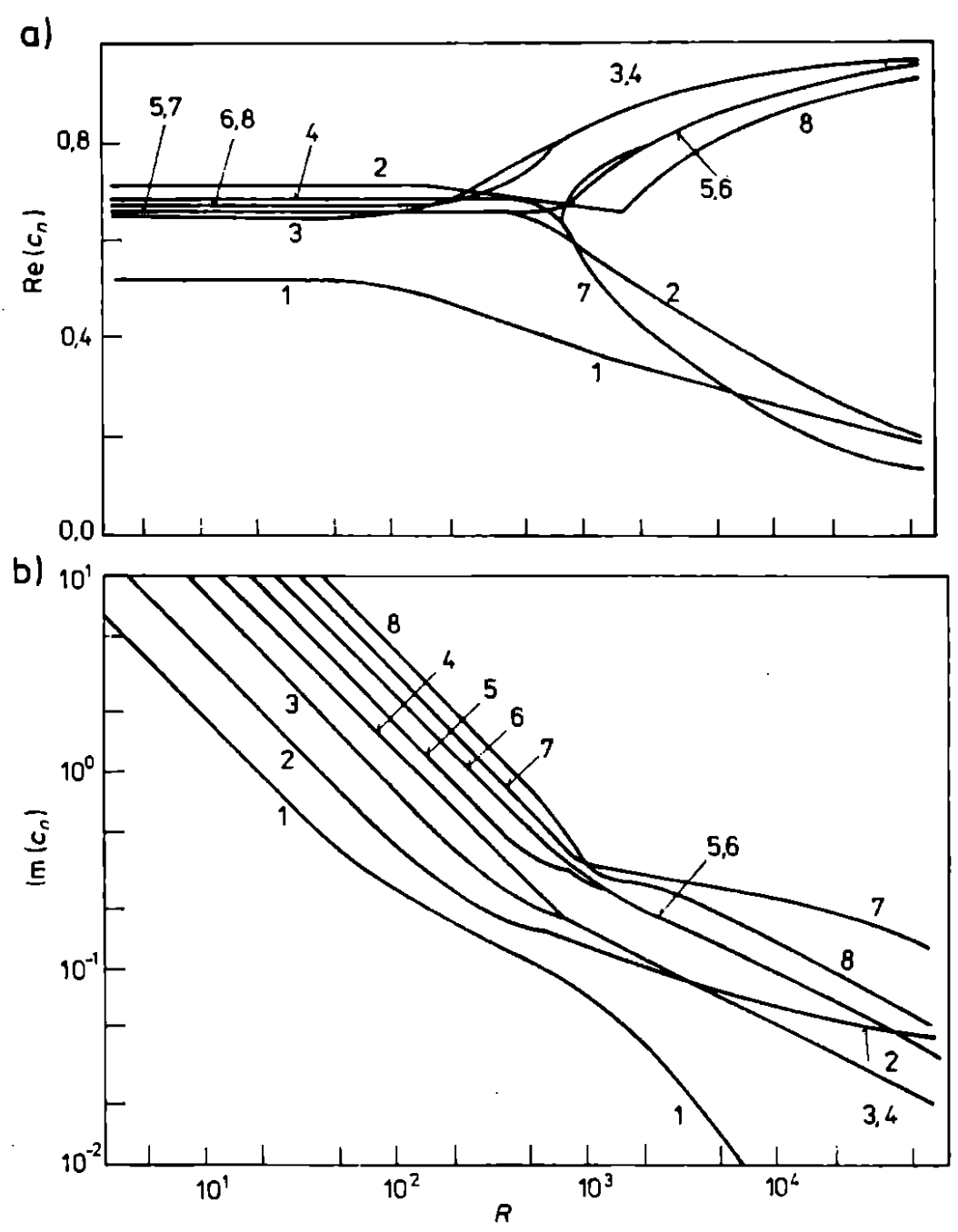

Fig. 3. a) Real parts of the first eight eigenvalues versus $R$ for $\alpha=2.0$. Note that ordering of the eigenvalues is by the magnitude of the eigenvalue at low $R$. b) Imaginary parts of the first eight eigenvalues versus $R$ for $\alpha=2.0$. Note that the crossings do not represent degeneracies since the real parts of the eigenvalues are not equal at the points where the imaginary parts are, and vice versa (Grosch and Salwen (1968))

in this case. Table 1 gives a set of the results he obtained, and we see that the approach to the limit is slow. The existence of a finite range of $R$ for which $c_{i}>0$, implying that this is an unstable basic flow, is interesting since Rayleigh's criterion establishes that the flow is stable to inviscid disturbances. Rayleigh's equation in fact does not have any solutions with $0<c_{r}<1$; the limit solution of Davey's calculation as $R \rightarrow \infty$ would have critical layers occurring on the boundaries.

The investigation of the solution of (2.12) for a Blasius flow in the range $0<y<\infty$ is especially interesting because it turns out that the "critical level" occurs in the interior of the flow field. As usual the shear flow and the Reynolds number are scaled by the displacement thickness. In Table 2 we 
Table 1

Principal eigenvalue $c$ for plane Poiseuille flow at various $R$ (Davey (1973))

\begin{tabular}{c|c}
\hline & Poiseuille \\
\hline $10^{4}$ & $0.2375+0.0037 i$ \\
$2.5 \cdot 10^{4}$ & $0.2012+0.0020 i$ \\
$5 \cdot 10^{4}$ & $0.1753-0.0053 i$ \\
$10^{5}$ & $0.1459-0.0150 i$ \\
$10^{6}$ & $0.0666-0.0140 i$ \\
\hline
\end{tabular}

give the values of $c$ as computed from (2.12) at $R=10000$ and those computed from (2.5) by deforming the path of integration in the complex $y$-plane below the real axis of $y$ (courtesy Davey (1978)). It is seen that where both sets of solution exist the two sets of values are very close, and since

Table 2

Values of $c$ computed from Rayleigh's equation and the Orr-Sommerfeld equation at $R=10000$ for Blasius flow (Davey (1978))

\begin{tabular}{c|c|c}
\hline & Rayleigh & Orr-Sommerfeld \\
\hline 0.1 & $0.219-0.010 i$ & $0.222+0.008 i$ \\
0.2 & $0.343-0.046 i$ & $0.246-0.042 i$ \\
0.3 & $0.424-0.094 i$ & $0.425-0.091 i$ \\
0.4 & $0.483-0.147 i$ & $0.483-0.145 i$ \\
0.5 & $0.530-0.203 i$ & unobtainable \\
0.8 & $0.672-0.398 i$ & unobtainable \\
1.0 & $0.831-0.519 i$ & unobtainable \\
1.3 & $1.088-0.598 i$ & unobtainable \\
\hline
\end{tabular}

$c_{i}<0$ the $y$-plane must be cut across the real axis in the solution to Rayleigh's equation. We note that even though $0<U<1$, it is possible for $c_{r}>1$ and this does not contradict Howard's semicircle theorem which depends on $\psi$ being smooth. Further, no solutions of the Orr-Sommerfeld equation were found for $x>0.48$ but the reason is not known.

The nature of the solutions when $R \gg 1$ was broadly discussed by Lin (1955) (see also Tatsumi and Gotoh (1969)) but it is appropriate to go into a little more detail about their features. Let us suppose that $c_{i}$ is small, but finite, when $R$ is large, and attempt to set up a uniformly valid leading term of the expansion of the solution in powers of $c_{i}$ for large $R$. We may reasonably expect that except in the neighbourhood of $y=y_{c}$, where $U\left(y_{c}\right)$ $=c_{r}$, this is provided by Rayleigh's equation. Thus as $y \rightarrow y_{c} \pm 0$.

$$
\psi \sim A_{ \pm}\left[\frac{U\left(y_{c}\right)}{\left(U^{\prime}\left(y_{c}\right)\right)^{2}}(U(y)-c)\left\{\log (U(y)-c)+B_{ \pm}\right\}\right],
$$


where $A_{ \pm}, B_{ \pm}$are constants differing on the two sides of $y=y_{c}$. Now if $B_{ \pm}$ are suitably related we may pass through $y=y_{c}$ smoothly without need to invoke viscosity and the eigenfunction of Rayleigh's equation is the limit of the eigenfunction of the Orr-Sommerfeld equation. This condition is

$$
B_{+}-B_{-}=\pi i \operatorname{sgn}\left(\frac{c_{i}}{U^{\prime}\left(y_{c}\right)}\right)+o(1)
$$

where $c_{i} \ll 1$. However, there are other constraints on $B_{ \pm}$due to the conditions on $\psi$ at $y=y_{1}, y_{2}$ and as we have seen it may not be possible to satisfy (2.15). Viscous effects must then be invoked in the neighbourhood of $y=y_{c}$ where we write

$$
a=U^{\prime}\left(y_{c}\right), \quad b=-U^{\prime \prime}\left(y_{c}\right), \quad \mu^{2}=\frac{\alpha R a^{4}}{b^{3}},
$$

$$
\beta=\frac{b c_{i}}{a^{2}}, \quad y=y_{c}+\frac{a}{b} Y,
$$

and without loss of generality we take $a>0, b>0$.

On expanding the various coefficients in (2.12) in powers of $Y$ and retaining only the leading terms we have

$$
\frac{d^{4} \psi}{d Y^{4}}-i \mu\left[(Y+i \beta) \frac{d^{2} \psi}{d Y^{2}}+\psi\right]=0
$$

and we are interested in the solution of (2.17) when $Y=O(\beta)$ in the limit $\mu \rightarrow \infty$ (Wasow (1948)). One solution can be excluded on the grounds of exponential growth as $Y \rightarrow \infty$ and two others reduce to solutions of Rayleigh's equation as $\mu \rightarrow \infty$. The additional solution may be written as

$$
\psi_{3}=\int_{C} \frac{1}{s^{2}} d s \exp \left\{\frac{-i}{s}-i(Y+i \beta) s-\frac{s^{3}}{3 \mu^{2}}\right\}
$$

where $\mathrm{C}$ is a contour in the $s$-plane beginning at $s=0$, moving along the positive imaginary axis of $s$ and then swinging round to end at infinity along the positive real axis of $s$. The value of $\psi_{3}$ when $\mu \gg 1$ is dominated by the contribution from the saddle at $s^{2}=-\mu i(Y+i \beta)$, with $\operatorname{Re} s>0$, provided it is exponentially large.

Let us first suppose $\beta>0$. Then the contribution from the saddle is exponentially large if $|Y|<\beta \sqrt{3}$ and

$$
\psi_{3} \sim \frac{\pi^{1 / 2}}{\mu^{3 / 2}(\beta-i Y)^{3 / 4}} \exp \left[\frac{2}{3} \mu(\beta-i Y)^{3 / 2}\right],
$$

whereas if $|Y|>\beta \sqrt{3}$ we may neglect the contribution from the saddle and replace the path of integration by one along the positive imaginary axis if 
$Y>0$ and denoted by $C_{1}$ in Figure 4, while if $Y<0$ we may use one which swings round to end along the negative imaginary axis (with $\operatorname{Re} s=0+$ ) and denoted by $C_{3}$ in Figure 4 . The values of the resulting integrals when $\mu=\infty$

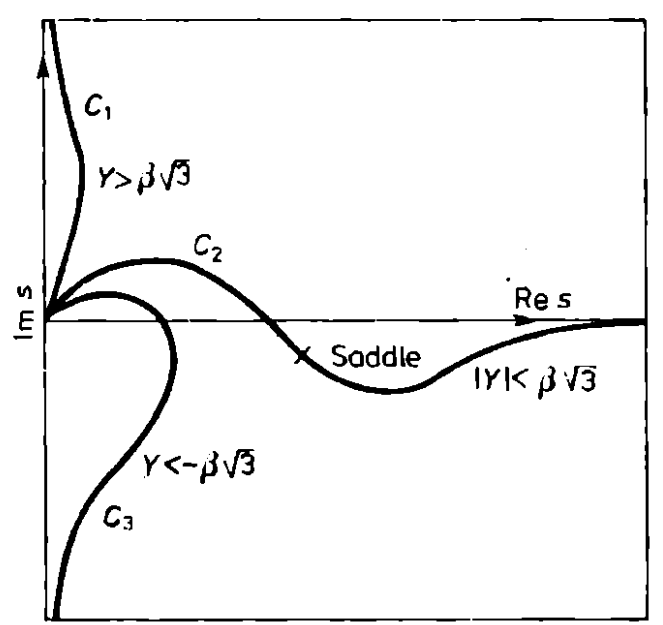

Fig. 4. Contours for the integral in equation (2.18)

are similar to (2.14) when $\beta \ll|Y| \ll 1$ but the sign of $B_{+}-B_{-}$in $(2.15)$ is changed. We thus have the flexibility to satisfy the requirement on $B_{+}, B_{-}$ provided by the conditions at $y_{1}, y_{2}$. Hence the solution of the OrrSommerfeld equation, when $R \gg 1$ and $\beta$ is small, is smooth if

$$
\left|y-y_{c}\right|>-\sqrt{3} \frac{c_{i}}{\left[U^{\prime}\left(y_{c}\right)\right]}
$$

and is violently oscillatory otherwise. An example is provided in Figure 5.

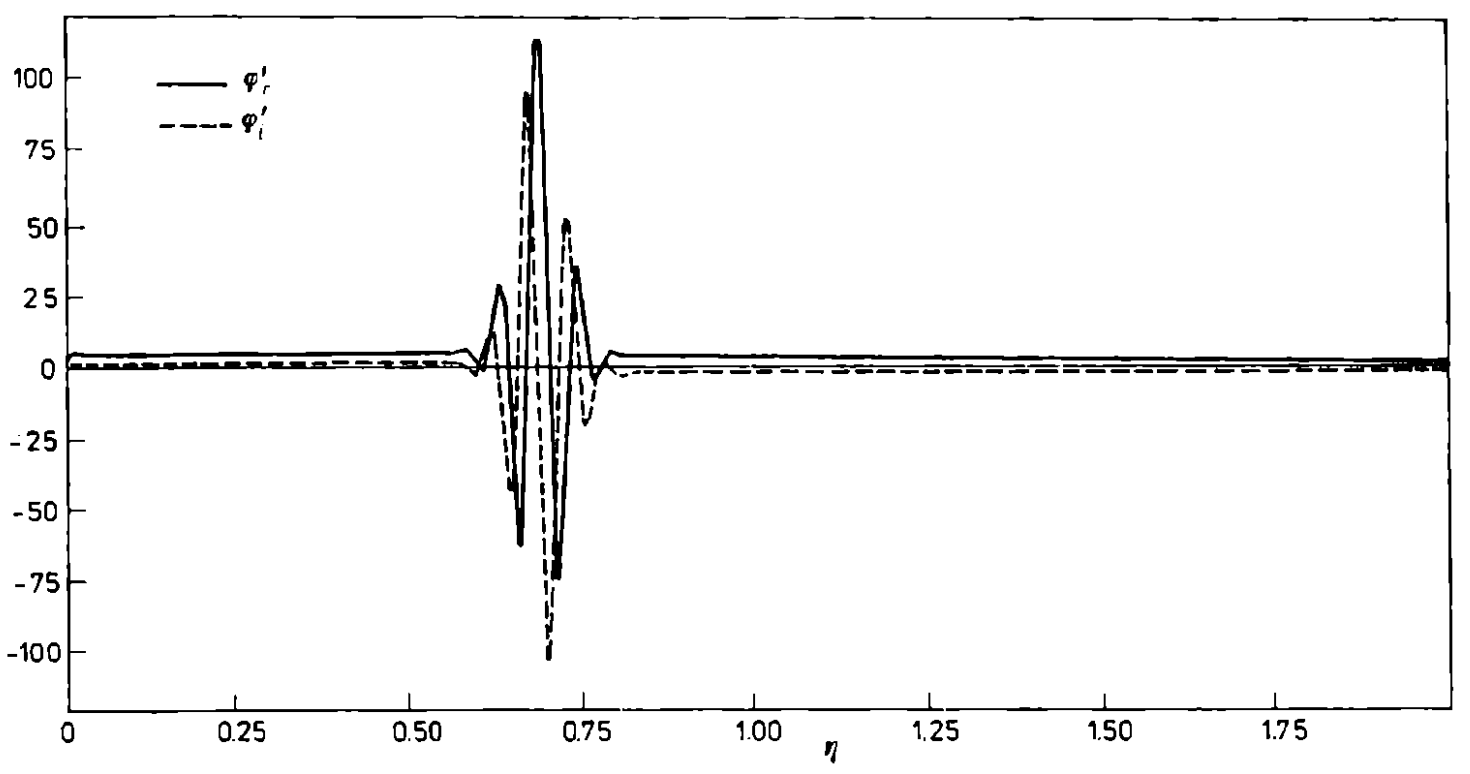

Fig. 5. Blasius eigenfunction for $x=0.179$ and $R=10^{6}, c=0.32165-0.03629 i$ (Davey (1978) 
Further, we may see the reason that the deformation of the contour in the complex $y$-plane below the real axis of $y$, in obtaining the solution of Rayleigh's equation, yields the correct limiting value of $c$ as $R \rightarrow \infty$ for the Orr-Sommerfeld equation. Once $\operatorname{Im} y<-\beta$ (2.19) becomes exponentially small and the acceptable solutions of the Orr-Sommerfeld equation become identical with those of the Rayleigh equation.

On the other hand, if $|\beta|<0$ the solution (2.19) is exponentially small when $Y$ is real and $|Y|<\beta \sqrt{3}$ so that $\psi_{3}$ remains smooth for all $Y$. Further the sign of $B_{+}-B_{-}$for this solution is the same as in (2.15); hence the difficulty mentioned above in connection with the Rayleigh solution of (2.12) cannot be eliminated. We recall that the difficulty must exists otherwise Rayleigh's equation would have an acceptable solution when $U^{\prime \prime}(y)<0$ in $y_{1}$ $<y<y_{2}$. The conclusion is that if the limit, as $R \rightarrow \infty$, of the eigenvalue $c$, computed from (2.12), is the same as that computed from (2.5) by contour deformation then

$$
c_{i}<0 .
$$

Thus if $U^{\prime \prime}$ does not change sign a direct integration of Rayleigh's equation does not yield a value to $c$ (expect by invoking non-linear effects as in the Benney-Bergeron theory which we shall consider later) but the value obtained after deforming the contour is the correct limit of the corresponding viscous problem as $R \rightarrow \chi$ provided that $c_{i}<0$. The two eigenfunctions are different, the one containing a discontinuity on the real axis of $y$ and the other a finite range $y_{3}<y<y_{4}$ in which $\psi$ oscillates infinitely. This range is specified accurately by $(2.20)$ if $c_{i}$ is small. In general Foote and Lin (1950) find that if $y_{0}$ is the point in the complex plane of $y$ at which $U(y)=c$, then $y_{3}, y_{4}$ are defined by the requirement that the real parts of

$$
\int_{y_{0}}^{y_{3}, y_{4}}[i(U-c)]^{1 / 2} d y
$$

are zero.

As Mack's computations in Figure 2 show, the conclusion $c_{i}<0$ in the limit $R \rightarrow \infty$ does not imply that the shear flow is necessarily stable for all $R>0$ but in the case of circular Poiseuille flow this appears to be the case. If the flow is unstable at finite values of $R$, the instability can persist for arbitrarily large values of $R$ manifesting itself in modes of increasingly long wave-length $(\alpha \rightarrow 0)$. There is an extensive literature on this subject, an important recent paper being due to Lakin, $\mathrm{Ng}$ and Reid (1978).

2.3. Unstable Flows. The violation of Rayleigh's criterion does not necessarily imply that the flow is unstable. For example if $U=\sin y, U^{\prime \prime}$ changes sign at $y=0$, but the flow is stable if $y_{2}-y_{1}<\pi$ (Drazin and Howard (1966), p. 35). Let us consider a class of shear profiles $U(y: \chi)$ where 
$\chi$ is some parameter characterizing the flow and such that $U^{\prime \prime}$ changes sign only if $\chi>0$. Then one may expect that instability occurs for $\chi>\chi_{c}$ where $\chi_{c}$ is positive or zero. Once it sets in there is a range of values of $\alpha,|x|<\alpha_{x}$ such that Rayleigh's equation has non -trivial solutions for some $c$. If we were to admit non-smooth profiles (which we explicitly shall not) it would be difficult to conjecture any general properties of $c$ and $\alpha_{x}$. but for smooth profiles it is quite possible that $\alpha_{y}$ is bounded and the number of values of $c$ for each admissible $\alpha$ is limited. For example, if $U=\operatorname{sech}^{2} y(-\infty<y<\infty)$ a sinuous mode has been found, in which $\psi$ is symmetric, if $|\alpha|<1$, and essentially one corresponding value of $c$ (another is its complex conjugate). In addition if $|\alpha|<2$ there is a varicose mode, in which $\psi$ is antisymmetric, and a different corresponding value of $c$. Each class of mode is terminated by a smooth solution in which $c$ is real and $U^{\prime}=0$ when $U^{\prime \prime}=c$. Indeed, the Reynolds' stress criterion (2.11) prevents $\psi$ from having a logarithmic singularity at $U=c$. It is conjectured that for all smooth monotonic profiles, or monotonic apart from a symmetry requirement, non-trivial $\psi$ exist for a finite range of $x$ only and at $x=\alpha_{\chi}, c$ is real and $\psi$ is smooth. The number of classes of solution may well be equal to the number of points of inflexion of the basic profile (Howard (1964)).

For other classes of smooth profiles $\alpha_{x}$ also appears to be bounded but it is less clear that the limit solution at $\alpha=\alpha_{y}$ is smooth. For example, if $U$ $=\operatorname{sech}^{2} y \tanh y$, two classes of solution have been found, one with $c_{r} \neq 0$ and known as a propagating mode, in which $c_{i}=0$ if $\alpha \simeq 1$ with a singularity at the critical level. The reason is that for this profile there are two critical values of $y$ at which Reynolds stress jumps occur but cancel. There is another, standing, mode in which $c_{r}=0$ and $c_{i}$ vanishes if $\alpha=5$. We identify this with $\alpha_{x}$ and note that the corresponding solution is smooth (Drazin and Howard (1966), p. 40). Another example is $U=e^{-y} \sin y, 0<y<\infty$ for which solutions have been computed if $0<x<0.78$, the solution for $\alpha=0.78$ appearing to have a logarithmic singularity at the (two) critical layer(s) (Gill and Davey (1969)). There may, however, be another class of solutions terminating smoothly at a different value of $\alpha$. No acceptable solutions have been reported for $\alpha>0.78$. Formally solutions may be looked for by deforming the path of integration below the real axis of $y$ in the manner described by Lin (1955) and as carried out explicitly for a more complicated equation by Lessen, Singh and Paillet (1974). For the profile $U$ $=e^{-v^{2}},|y|<\propto$, McKay (1979) has found values of $c$ at larger values of $\alpha$ than that for which $c$ is real. However, the sign of $c_{i}$ implies that when the contour is deformed back to the real axis of $y, \psi$ then is discontinuous at some value of $y$, due to the presence of a cut across this real axis. Were we considering the Rayleigh equation as a possible limit of the Orr-Sommerfeld equation as the Reynolds number $R \rightarrow \infty$, the associated limit solution would be a solution of the Rayleigh equation except in a finite range of values of $y$. In this range $\psi$ would oscillatc infinitely. 
Returning to consideration of the parameter $\chi$, we examine the nature of the perturbation in a state of marginal instability when $\chi-\chi_{c}$ is small and positive. This situation is attractive to study, from an evolutionary standpoint, since the instability develops sufficiently slowly that the effects of nonlinearity, viscosity and other physical parameters may be discussed in an orderly way. For larger values of $\chi$ the instability develops too rapidly to enable us to keep track of its properties at present. In marginal stability the critical mode has $\alpha=0$ for a wide class of shear flows. For if such a mode exists with $\alpha \neq 0$ and $U^{\prime \prime} /(U-c)$ does not change sign for this mode then (Howard and Drazin (1966)) there are unstable modes at smaller values of $\alpha$. Lin (1945) has given a formula for calculating the growth rates of neighbouring modes. On the other hand, the critical mode may well have $\alpha \neq 0$ in specific instances. Howard and Drazin (1964) have given such an example in the closely related problem of Rossby waves. In all examples of marginal stability known to the author the critical mode has a smooth eigenfunction and it is quite likely that singular modes can occur only if the basic flow is definitely unstable.

2.4. The initial-value problem. The discussion of stability of plane parallel flows has so far been based on (2.4), i.e., on the assumption that a simple seperation-of-variable argument is adequate. A more satisfactory approach, in which (2.4) can be embedded, is to consider a small initial disturbance to the basic flow and examine how it evolves with time. Instability may thus be defined as a solution in which the disturbance does not remain small for all time. The procedure now is to solve (2.2) using a Fourier transform with respect to $x$, and a Laplace transform with respect to $t$, whereupon the transformed independent variable $\psi$, of $\psi$, satisfies (2.5), with a specified right-hand side depending on the precise nature of the initial conditions. The normal mode analysis based on (2.4) may then be interpreted as identifying the poles of $\bar{\psi}$ and if they lie above the real axis of $\omega$ instability results. They are, however, not necessarily the only indication of instability. Contributions to $\psi$ also come from any branch points of $\bar{\psi}$ and these are algebraic in nature being $O\left(t^{-n}\right)$ as $t \rightarrow \infty$, where $n$ has to be found. For any $n$, derivatives of $\psi$ with respect to $y$ of sufficiently high order become unbounded as $t \rightarrow \infty$ due to the contributions from these branch points. A reasonable requirement for stability is that $n>1$ in which case the pressure gradients induced by the non-linear terms decay to zero as $t \rightarrow \infty$.

The complete solution of the non-homogeneous form of (2.5) in any particular case is clearly a formidable task and has been the subject of some controversy (see Brown and Stewartson (1980)). Some of the principal features of the branch singularities may, however, be seen from a study of Couette flow, in which $U=y$ and (2.3) reduces to

$$
\left(\frac{\partial}{\partial t}+y \frac{\partial}{\partial x}\right)\left(\frac{\partial^{2} v}{\partial x^{2}}+\frac{\partial^{2} v}{\partial y^{2}}\right)=0
$$


with a first integral

$$
V_{1}^{2} v=\frac{r^{2} v}{\partial x^{2}}+\frac{\partial^{2} v}{r^{2}}=F(x-y, y)
$$

where $F$ is the given initial value of $\Gamma_{1}^{2} v$. Provided that $F$ has compact support, i.e., vanishes outside a finite interval of $x$, it follows that when $t$ is large

$$
v \sim t^{-2} G(x-y t, y) \quad \text { where } \frac{\hat{c}^{2} G}{\partial x^{2}}=F .
$$

Thus $n=2$ and all velocity components decay as $t \rightarrow x$, showing that the basic flow is stable. A variation of this initial value problem is to suppose that $v=0$ at $y_{1}, y_{2}$ only for $t>t_{0}$ where $t_{0}$ is a positive constant. An example is provided by the problem of long Rossby waves a uniform shear. The governing equation is

$$
\left(\frac{\partial}{\partial t}+y \frac{\partial}{\partial x}\right) \frac{\hat{r}^{2} v}{\partial y^{2}}+\beta \frac{\partial t}{\partial x}=0
$$

(Dickinson (1970); Warn and Warn (1976)) with $\beta$ constant and $y_{2}>0$, $y_{1}=\sigma x$. Let us suppose that $u^{\prime}\left(y_{2}\right)=e^{i x x}$ for $t_{0}>t$ and $v\left(y_{2}\right)=0$ for $t>t_{0}$. Then

$$
v=\frac{1}{2 \pi i} \int_{\gamma-i x}^{\gamma+i x} d s \frac{1-e^{-s t_{0}}}{s} \sqrt{\left.\frac{i s-x y}{i s-x y_{0}} \frac{K_{1}\left(2 \sqrt{K_{1}(2 \sqrt{1 i s / x-y)})}\right.}{\beta\left(i s / x-y_{0}\right)}\right)} e^{s t+i x x}, \quad i>0
$$

where $K_{1}$ is a Bessel function, and it may be deduced from the properties of the integral (2.27) that $t \sim t^{-2}$ as $t \rightarrow x$. We infer that in general $t \sim t^{-2}$ also so long as the forcing from the boundaries has compact support both in time and space.

Without the condition of compact support much less can be said. Thus if $F \equiv 1$ then $v=-\frac{1}{2}\left(y_{2}-y\right)\left(y-y_{1}\right)$ and remains finite for all $t$. In the study of non-linear effects on which we shall now embark the initial disturbance will always be thought of as centred and vanishing outside a certain distance from the origin. Thus, according to linear theory, at large times the algebraic components of the disturbance decay like $t^{-2}$ and it will only be necessary to consider the behaviour of the modal oscillations, which in a state of marginal instability, are slowly growing in amplitude.

\section{Evolution of forced critical layers}

3.1. Introduction and linear theory. In order to focus attention on the critical layer we consider forced oscillations of a prescribed wave-length and period. As a result $c$ is a fixed real velocity and the existence of a definite 
value of $y$ at which $U(y)=c$ is assumed. We are then able to consider how any singularity there may evolve under the influence of non-linear and viscous effects without having to take account of the possibilities either that this critical layer does not exist because of the overall constraints on the disturbance, or that the corresponding disturbance is the least important of all possible modal disturbances as would generally be the case with free oscillations. The treatment of unforced oscillations is delayed until the next section.

Although the restriction is not strictly necessary the neatest way of discussing the evolution of the critical layer is through the Rossby equation describing quasi-geostrophic motions in a region of the atmosphere bounded by lines of latitude. The governing equations have been written down by Pedlosky and Greenspan (1967), using the sliced cylinder as an example, and for weakly viscous disturbances forced by an oscillation of long wavelength at a fixed latitude on a constant shear flow they may be reduced to the form

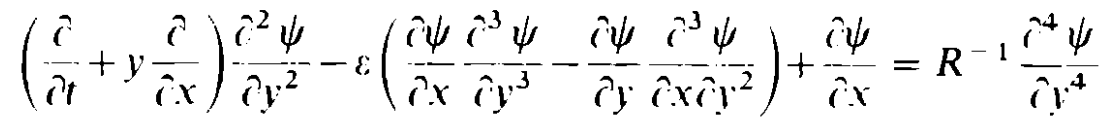

(Stewartson (1978)). Here $\psi$ is the reduced stream function of the disturbance, $x$ and $y$ are non-dimensional measures of distance in the eastward and northward directions, $\varepsilon(\ll 1)$ is a measure of the amplitude of the disturbance, and $R(\gg 1)$ is a Reynolds number in which the length scale partly depends on the wavelength of the imposed oscillation. Also the origin is moving in the $x$-direction with the phase of the disturbance so that $y=0$ is the (fixed) critical level. The forcing is switched on at $y=y_{0}(>0)$ when $t=0$ and we are interested in the evolution in $-\infty<y<y_{0}$ for all time. The boundary and initial conditions which we use are

$$
\begin{aligned}
& \psi=\cos x \text { at } y=y_{0} \quad \text { for } \quad 1>0 \text {, } \\
& \psi \rightarrow 0 \quad \text { as } y \rightarrow-\infty \text { for } t>0 \text {, } \\
& \psi=0 \quad \text { for } y \leqslant y_{0} \quad \text { and } t=0 \text {. }
\end{aligned}
$$

Strictly, another condition is required at $y=y_{0}$. e.g., no slip, but since $R \gg 1$ the violation of such a condition is of little consequence. The use of a (radiation) condition at $!=-x$ is simply to avoid the additional complication of reflection from a lower boundary. Another way of looking at the limiting process involved in obtaining (3.1), (3.2) is that it enables us to examine the properties of a critical layer in a uniform shear and to neglect $\alpha^{2} \psi$ in comparison with $\psi^{\prime \prime}$ in (2.5).

Provided $\psi$ remains finite we may neglect the terms proportional to $\varepsilon$ and $R^{-1}$ in (3.1) and then at large times $\psi$ takes the form (Dickinson (1970); Warn and Warn (1976))

$$
\psi \begin{cases}A(x, t)|y|^{1 / 2} K_{1}\left(2|y|^{1 / 2}\right) & \text { for } y<0 \\ y^{1 / 2}\left[B(x, t) J_{1}\left(2 y^{1 / 2}\right)+C(x, t) Y_{1}\left(2 y^{1 / 2}\right)\right] & \text { for } y>0\end{cases}
$$


where $J_{1}, Y_{1}, K_{1}$ are Bessel functions of order unity and $A, B, C$ are functions of $x$ and slowly varying functions of $t$, to be found. By slowly varying we mean that $t$ appears in the form $\varepsilon^{n} t$ or $R^{-m} t$ where $n, m>0$. Two relations connecting $A, B, C$ are immediate, namely

$$
B J_{1}\left(2 y_{0}^{1 / 2}\right)+C Y_{1}\left(2 y_{0}^{1 / 2}\right)=y_{0}^{-1 / 2} \cos x
$$

from (3.2) and

$$
\pi A+C=0
$$

from the reasonable requirement that $\psi$ be continuous across $y=0$. A third condition is required, to fix the solution, for which we must investigate the structure of the critical layer near $y=0$. If non-linear terms are neglected then this condition may be obtained by considering the growth of the critical layer with $R=\infty$ or by supposing that the solution has settled down in some sense, either argument leading to the same result as we shall now demonstrate.

From (3.3)

$$
\psi= \begin{cases}A(x, t)[1+|y| ; \log |y|+2 ;-1\}]+o(y) & \text { as } y \rightarrow 0^{-} \\ A(x, t)[1-y ; \log y+2 ;-1\}]+B y+o(y) & \text { as } y \rightarrow 0^{+}\end{cases}
$$

where $\gamma$ is Euler's constant. In order to look at the structure of the critical layer from the simplest evolutionary standpoint we set $\varepsilon=R^{-1}=0$ in (3.1) and assume that $\gamma \psi / \partial x$ is virtually constant across the critical layer at $y=0$. Then when $t \gg 1$ and $y \sim 1$

$$
\left(\frac{\partial}{\partial t}+y \frac{\partial}{\partial x}\right) \frac{\partial^{2} \psi}{\partial y^{2}}=-A^{\prime}(x, 0), \quad A^{\prime}=\frac{\partial A}{\partial x}
$$

so that

$$
\frac{\hat{c}^{2}}{\hat{c}^{2}} \cdot \frac{\psi}{2}=\frac{A(x-y t, 0)-A(x, 0)}{y}
$$

since $A(x, t)$ is supposed to be a slowly varying function of $t$. The match of (3.8) with (3.6) as $|y t| \rightarrow x$ and $y \rightarrow 0$ is now complete provided

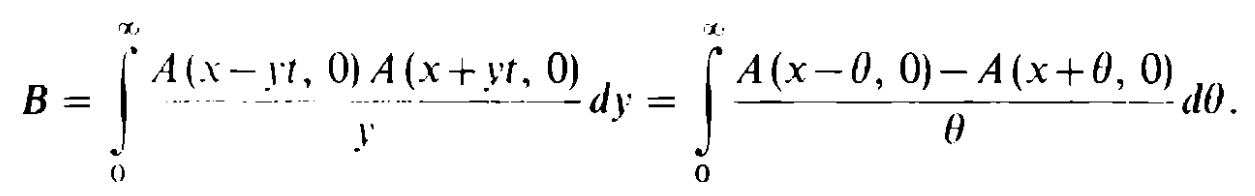

This condition, in which $t$ does not explicitly appear, completes the specification of (3.3). It is easy to see in fact that if $A$ is sinusoidal and we write

$$
A=\operatorname{Re} A_{0} e^{i x}, \quad B=\operatorname{Re} B_{0} e^{i x},
$$

where $A_{0}, B_{0}$ are complex constants, then

$$
B_{0}+i \pi A_{0}=0, \quad \pi A_{0}\left[Y_{1}\left(2 y_{0}^{1 / 2}\right)+i J_{1}\left(2 y_{0}^{1 / 2}\right)\right]=-y_{0}^{1 / 2},
$$


corresponding to the well-known phase jump of linear theory referred to in (2.9). It should be noticed that the thickness of the critical layer in this argument is $O\left(t^{-1}\right)$ and that the non-linear terms of (3.1) may no longer be neglected when $\varepsilon t^{2} \sim 1$. Thus, more strictly, $A$ is of the form $A\left(x, \varepsilon^{1 / 2} t\right)$ when $\varepsilon^{1 / 2} t \sim 1$.

Again, in order to look at the structure from the simplest steady but dissipative standpoint we set $\varepsilon=0, \partial / \partial t=0$ and take $R \gg 1$. Then (3.7) is replaced by

$$
R^{-1} \frac{\partial^{4} \psi}{\partial y^{4}}-y \cdot \frac{\partial^{3} \psi}{\partial x \partial y^{2}}=A^{\prime}(x, 0)
$$

This equation may be solved in a formal fashion but we anticipate that it leads to the solution (3.6) except when $|y| \ll 1$ and $\psi$ then satisfies.

$$
R^{-1 / 3} \frac{\partial^{2} \psi}{\partial y^{2}}=-\operatorname{Re} i A_{0} \int_{0}^{x} \exp \left\{-\frac{1}{3} u^{3}+i x-i y u R^{1 / 3}\right\} d u,
$$

(Tietjens (1925)). A match with (3.6) is again complete provided

$$
B_{0} e^{i x}=-2 i A_{0} e^{i x} \int_{0}^{x} \frac{\sin y u}{u} d u=-\pi i A_{0} e^{i x},
$$

as in (3.10). The thickness of the critical layer is now $O\left(R^{-1 / 3}\right)$ and the vorticity is $O\left(R^{1 / 3}\right)$ inside it. Non-linear terms may therefore not be neglected in the critical layer if $\varepsilon R^{2 / 3} \geqslant 1$.

These arguments suggest that the evolution of the critical layer when non-linear effects and viscosity are of equal importance may be described by using a scaled set of variables, viz.

$$
y=Y \varepsilon^{1 / 2}, \quad \lambda=\left(R \varepsilon^{3 / 2}\right)^{-1}, \quad t=\varepsilon^{-1 / 2} \tau,
$$

$$
\frac{\partial^{2} \psi}{\partial y^{2}}=\varepsilon^{-1 / 2} \zeta(x, \tau, Y)
$$

On substituting these new variables into (3.1) and then setting $R \rightarrow x_{i},:=0$, we find that $\zeta$ satisfies

$$
\left(\frac{\partial}{\partial \tau}-Y \frac{\hat{c}}{\partial x}\right) \zeta-\frac{\partial A}{\partial x} \frac{\partial \hat{\zeta}}{\partial Y}+\frac{\partial A}{\partial x}=\lambda \frac{\partial^{2} \zeta}{\partial Y^{2}}
$$

since $\psi$ does not change across the critical layer to leading order. The match with (3.6) is complete provided

$$
B(x, \tau)=\int_{-x}^{* x} \zeta(x, \tau, Y) d Y
$$


where the ${ }^{*}$ denotes that the finite part is to be taken. The behaviour of $\zeta$, and hence of $A$ and $B$, is now investigated as a function of $x, \tau, y$ and $\lambda$.

3.2. Non-linear inviscid theory. Even when $i=0$ the solution of (3.15) is a formidable task because $A(x, t)$ has to be determined, simultaneously with $\zeta$, through $B$ and (3.4). A special case which may be fully solved arises if $J_{1}\left(2 y_{0}^{1 / 2}\right)=0$ when $A$ is proportional to $\cos x$ and without loss of generality we may then set

$$
A=-\cos x \text {. }
$$

Three procedures are now available. First we can expand $\zeta$ as a power series in $\tau$ whose coefficients are functions of $Y, \tau$ and $x$, in the form.

$$
\begin{aligned}
\zeta=\frac{1}{2} i\left[\zeta_{10}(Y, \tau) e^{i x}-\check{\zeta}_{11} e^{-i x}\right]-\frac{1}{4}\left[\check{\zeta}_{20} e^{2 i x}-\check{\zeta}_{21}+\check{\zeta}_{22} e^{-2 i x}\right]- \\
-\frac{1}{8} i\left[\check{\zeta}_{30} e^{3 i x}-\zeta_{31} e^{i x}+\check{\zeta}_{32} e^{-i x}-\check{\zeta}_{33} e^{-3 i x}\right]+\ldots,
\end{aligned}
$$

where $\check{\zeta}_{m n}=O\left(\tau^{m}\right)$. On substituting this form into (3.15) and solving for the $\check{\zeta}_{s}$ in sequence we obtain

$$
\begin{gathered}
\check{\zeta}_{10}=\int_{0}^{\tau} e^{-i Y \tau_{1}} d \tau_{1}, \quad \zeta_{20}=i \int_{0}^{\tau} e^{-2 i Y\left(\tau-\tau_{1}\right)} d \tau_{1} \int_{0}^{\tau_{1}} \tau_{2} e^{-i Y \tau_{2}} d \tau_{2}, \\
\zeta_{21}=\int_{0}^{\tau} d \tau_{1} \int_{0}^{\tau_{1}} \tau_{2} e^{-i Y \tau_{2}} d \tau_{2}+\int_{0}^{\tau} d \tau_{1} \int_{0}^{\tau_{1}} \tau_{2} e^{i Y \tau_{2}} d \tau_{2}
\end{gathered}
$$

and, in general, $\zeta_{m n}$ is given by an $m$-dimensional integral.

The value of $B$ follows from (3.16) on using the properties of $\delta$-functions. The necessary condition for $\zeta_{m n}$ to make a contribution to $B$ is that the hyperplane, on which the exponent in the $m$-dimensional integral vanishes in the $\tau_{1}, \tau_{2}, \ldots, \tau_{m}$ space, must contain points interior to the simplex $0 \leqslant \tau_{1} \leqslant \tau_{2} \leqslant \ldots \leqslant \tau_{m} \leqslant \tau$. The explicit evaluation of the successive terms is in principle straightforward but rapidly becomes tedious because of the number of polynomial integrals involved. We obtain

$$
B=-\pi \sin x+\frac{1}{192} \pi \tau^{4} \sin x-\frac{1}{5760} \pi \tau^{6} \sin 2 x+\ldots
$$

Eventually all the harmonics of $\sin x$ appear, the coefficient of $\sin n x$ having a leading term proportional to $\tau^{2 n+2}$ if $n>1$. The presence of these harmonics makes the nomenclature "phase jump" unsatisfactory in connection with the critical layer and it is preferable to refer to $B$ as a "velocity jump" the critical layer thus acting as a slip surface.

The second procedure is to note that, if we write

$$
u^{2}=Y^{2}+4 \sin ^{2} \frac{1}{2} x,
$$


$\zeta$ satisfies a first order wave equation in $x, \tau$ along contours $u=$ constant. These contours are closed if $u \leqslant 2$ and form the interior of Kelvin cat's eyes (Fig. 6). Consequently (O`Neil (1978)) a formal solution for $\zeta$ for all times

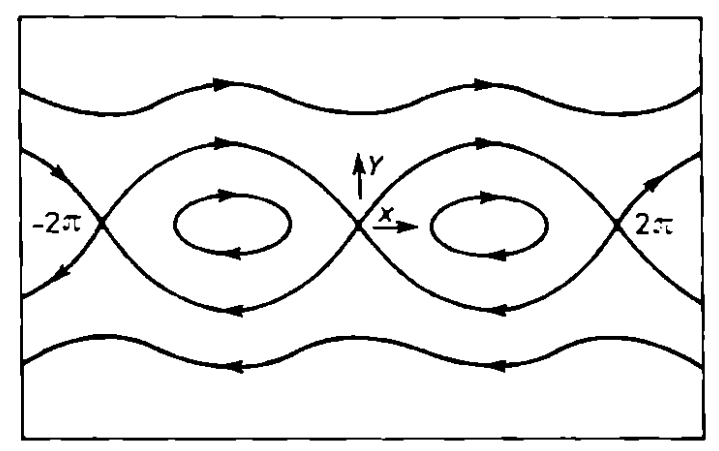

Fig. 6. Kelvin cat's eyes

may easily be written down in terms of elliptic functions. For example if $0<x<2 \pi, 0<\|<2$,

$$
\zeta=Y-u \operatorname{sgn} Y \operatorname{cn}\left(\varphi-\tau \operatorname{sgn} Y ; \frac{1}{2} u\right),
$$

where

$$
\sin \frac{1}{2} x=\frac{1}{2} u \operatorname{sn}\left(\varphi ; \frac{1}{2} u\right), \quad|Y|=u \operatorname{cn}\left(\varphi ; \frac{1}{2} u\right) .
$$

Whereas (3.20) is only useful for $\tau<4,(3.22)$ may be used to compute properties of $B$ for all time. In Figure 7 we display the variation of the coefficients of the first and second harmonics of $B$ as functions of $\tau$. and in Figure 8 the variation of $B$ itself at $\tau=0,2,6,10$. As $\tau$ increases, $B$ continues to oscillate, the period, however, being a function of $x$ varying from $2 \pi$ at $x=0$ to $x$ at $x=\pi$. Thus, at a fixed large value of $\tau$. $B$ is a rapidly oscillating function of $x$ with an amplitude which slowly decreases as $\tau \rightarrow x$. The upshot is that, at large values of $\tau, \zeta$ is developing pathological tendencies in the critical layer but the effect on the flow outside may well be insignificant, since its integrals with respect to $x$ and $y$ both tend to zero as $\tau<x$.

A third procedure, due to Warn and Warn (1978), is to solve (3.15) numerically with $\lambda=0$ by using a Fourier series representation in $x$ truncated to 55 terms, a fourth-order accurate finite difference scheme in $Y$ for $|Y| \leqslant 10$ and a leap-frog method to advance the system in time. Due to the appearance of small-scale noise of intorelable intensity this method can only be used for $\tau<\sim 4$ but it does have the advantage that the condition (3.4) may be incorporated, in the form

$$
A+r B=2 \pi \sin x,
$$

where $r$ is a constant. A change in the scale of $Y$ and a shift of $\pi / 2$ in the origin of $x$ is necessary for a complete identification of (3.4), (3.17) and (3.23). 


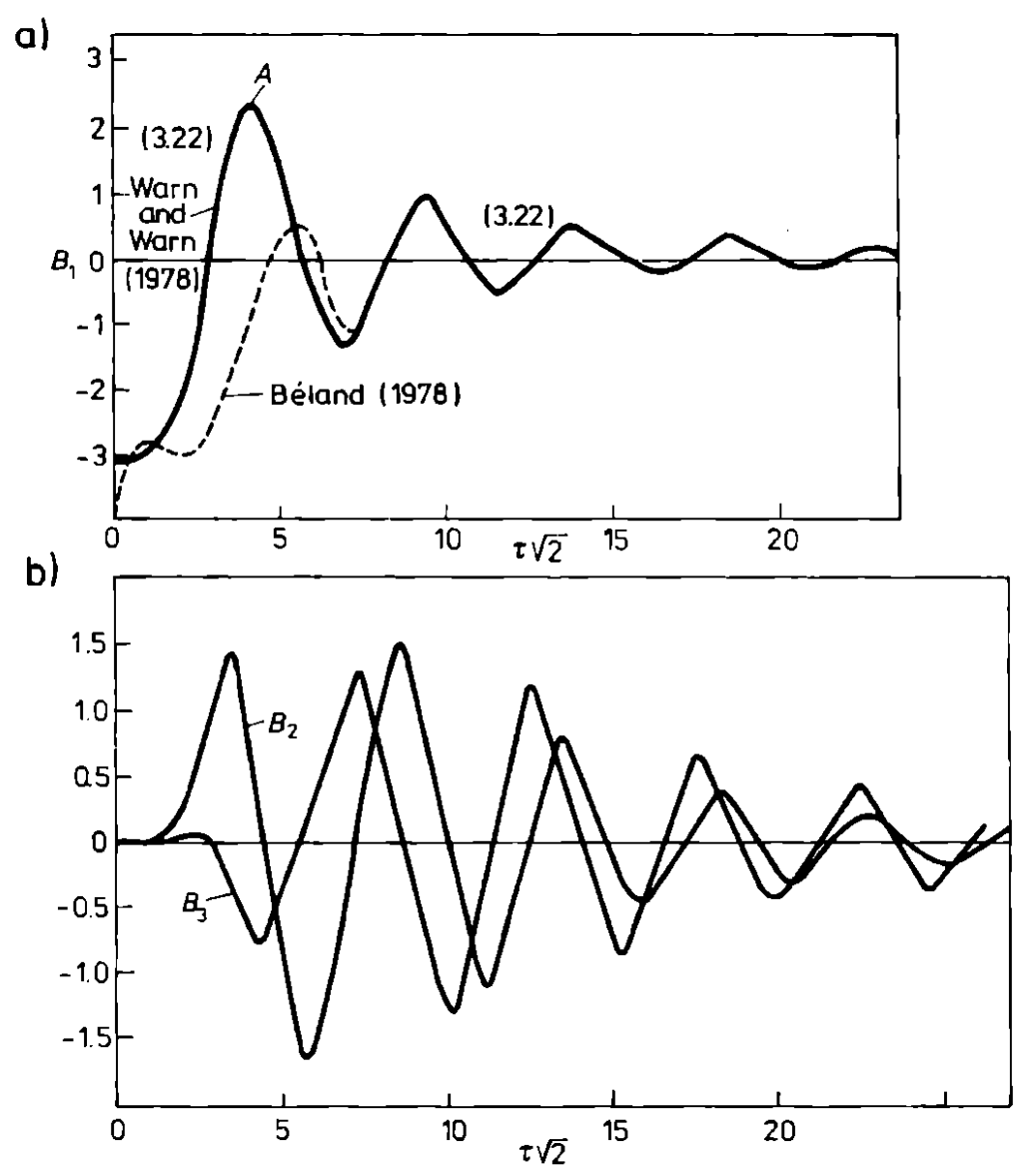

Fig. 7. a) Variation of $B_{1}$ with $\tau: B=\sum B_{n} \sin n x$. Warn and Warn's calculations terminate at $A$. b) Variation of $B_{2}$ and $B_{3}$ with $\tau$ (see Fig. 7a))

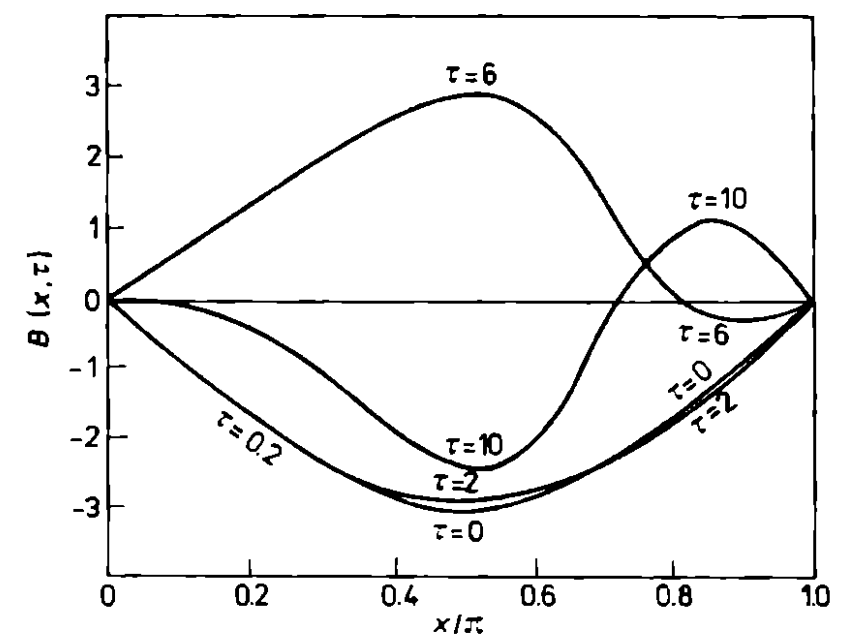

Fig. 8. Variation of velocity jump $B(x, \tau)$ across the critical layer with $x$ at $\tau=0,2.6,10$ when $A=-\cos x ; B$ is an odd function of $x$ with period $2 \pi$ 
The first method may also be used for general values of $r$, significant changes in (3.20) only occurring beyond the stage explicitly given. The second method may only be used for $r=0$. Warn and Warn carry out numerical studies for $r=0,0.10$ and 0.25 . The accuracy of their work is confirmed by the perfect agreement with the exact solution at $r=0$ shown in Figure 7, and for larger values of $r$ the most notable feature is the distoring of the cat's eyes, their centres being displaced to the left. In Figure 9 we display graphs of $\zeta$ at

a)

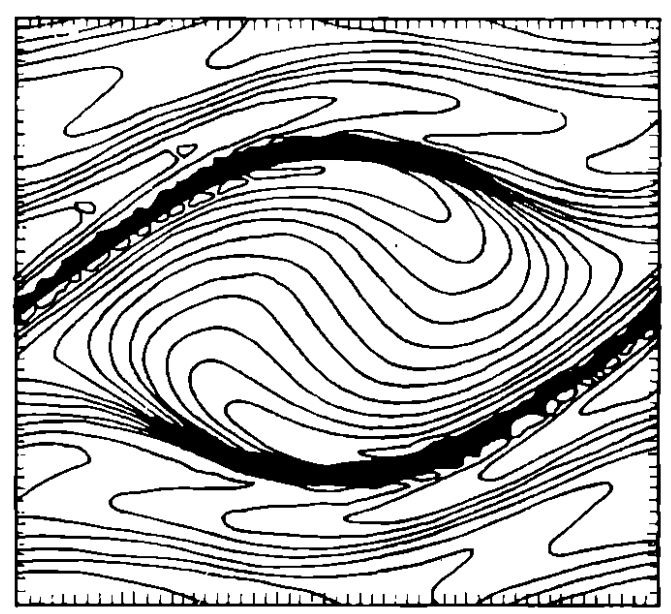

b)

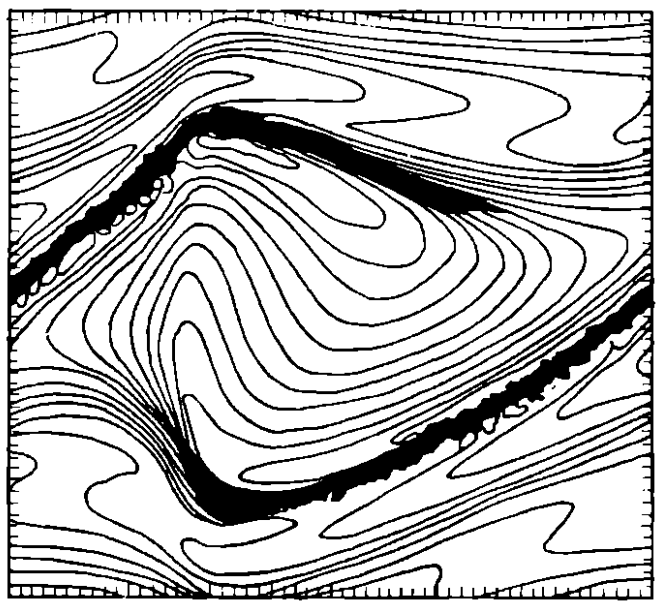

Fig. 9. Graphs of lines of constant ̌̈ (Warn and Warn (1978)): a) $r=0, \tau=4.25:$ b) $r=0.25$, $\tau=3.9$

$\tau=4.25$ for $r=0$ and at $\tau=3.9$ for $r=0.25$. It is interesting to note that even with the exact solution available great difficulty was found in drawing comparable graphs at larger values of $\tau$ (Rosen (1978)).

3.3 Non-linear viscous theory. No matter how small $\lambda$ is, must be expected that the increasingly pathological form of $\zeta$, especially inside the cat's eyes, will provoke viscous damping and eventially we can expect that the solution will become steady and the dependence on $\tau$ disappear. From (3.15) we might expect that this occurs when $\tau>i^{-1}$, i.e., when $t>R i$. There is a physical value in studying the final steady state in the critical layer because it is set up in a time much smaller than that $(\sim R)$ taken by the basic flow to disappear by diffusion.

It turns out to be sufficient to assume that (3.17) holds (i.e., $r$ is effectively zero) so that $\zeta$ satisfies

$$
Y \frac{\partial \zeta}{\partial x}-\sin \frac{\partial \zeta}{\partial y}+\sin x=\lambda \frac{\partial^{2} \zeta}{\partial y^{2}}
$$

and is periodic in $x$ with period $2 \pi$. A remarkable feature of $(3.24)$ is that if $\lambda$. 
is finite no solution can be found for which $\zeta \rightarrow 0$ as $|Y| \rightarrow \infty$ and at the very least we must require instead that

$$
\check{\zeta} \rightarrow H(i) \operatorname{sgn} Y, \quad \text { as } \quad Y \rightarrow \propto
$$

where $H$ is a constant for each $\lambda$. From direct integration of (3.15)

$$
H(\lambda)=-\frac{1}{4 \pi \lambda} \int_{0}^{2 \pi} B \frac{\partial A}{\partial x} d x=-\frac{1}{4 \pi \hat{\lambda}} \int_{0}^{2 \pi} B \sin x d x
$$

(Haberman (1972); Brown and Stewartson (1978)). This is a remarkable result for it implies that when the critical layer has settled down to a steady form its principal effect is to generate a discontinuity in the shear of the basic flow of a larger order of magnitude $\varepsilon^{1 / 2} H(\lambda)$, than that of the original disturbance. The velocity jump is only $O(c)$ and hence weaker. Since the original ideas on steady non-linear critical layers were written down by Benney and Bergeron (1969) and Davis (1969), there have been a number of applications most of which are in error due to neglect of this feature.

When $i \gg 1$ the solution may be expanded in descending powers of $i^{2 / 3}$, whose coefficients are functions of $x$ and $\lambda^{1 / 3} Y$, of which the leading term (3.13) was given by Tietjens (1925). Further terms may be computed in a straightforward but increasingly tedious way, and we have

$$
B=-\pi \sin x+0.511 \pi \lambda^{-4 / 3} \sin x-0.035 \pi \lambda^{-2} \sin 2 x+O\left(\lambda^{-8 / 3}\right)
$$

(Haberman (1976); Brown and Stewartson (1978)). Again, higher harmonics of $\sin x$ appear, as the expension is continued, indicating that the notion of a phase jump is inappropriate once non-linear effects are significant. Hence we have, from (3.26), that

$$
H(\lambda)=\frac{\pi}{4 \lambda}\left(1-0.511 \lambda^{-4 / 3}+\ldots\right)
$$

On restoring the time dependence in addition to viscous effects we find that the contribution, independent of $x$, to $\zeta$ has a leading term

$$
\frac{1}{2 \hat{\lambda}} \int_{0}^{\tau} \frac{\sin u z}{u}\left(e^{-u^{3 / 3}}-e^{2 u^{3 / 3}-u^{2} \tau}\right) d u, \quad z=Y \lambda^{1 / 3},
$$

showing that a diffusion layer is spreading out from the centre of the critical layer. This layer in fact acts as source of vorticity which reaches a distance $y$ from its centre in a time $t \sim R y^{2}$. Thus its full impact on the basic flow is achieved in a time comparable with that of any diffusive tendency of the main shear. (Only in Couette-Poiseuille flow is this tendency absent.) At 
small values of $\lambda$ we largely follow the argument given by Benney and Bergeron (1969) and write

$$
\zeta=\zeta_{0}(x, Y)+\hat{\zeta}_{1}(x, Y)+O\left(\lambda^{2}\right)
$$

After substituting this expansion into (3.24) we find that

$$
\zeta_{0}=Y+K(v), \quad \text { where } v=Y^{2}-4 \cos ^{2} \frac{1}{2} x
$$

and $K$ is a function of $v$ to be determined. It is found by writing down the equation for $\zeta_{1}$, and expressing the fact that $\zeta_{1}$ is periodic in $x$ with period $2 \pi$. This is only possible when $t>0$ if

$$
K^{\prime}(v)=A+\int_{0}^{2 \pi}\left(v+4 \cos ^{2} \frac{1}{2} x\right)^{1 / 2} d x
$$

for $Y>0$ with a similar result for $Y<0$. When $v<0$, (3.32) must be modified by replacing the limits of integration with the zeroes of $v$ $+4 \cos ^{2}(x / 2)$ and hence either $K$ is a constant or $\zeta$ has a logarithmic singularity at $x=0, r=-4$. The constants $A$. are fixed by the requirement of a bounded $\zeta$ as $|Y| \rightarrow x$ and are equal to $\mp \pi \operatorname{sgn} Y$. Thus

$$
\left.K(v)=B \cdot \mp \pi i_{0}^{n} d v_{1} \int_{0}^{2 \pi}\left(v_{1}+4 \cos ^{2} \frac{1}{2} x\right)^{1 / 2} d x\right\}^{1-1}
$$

according as $Y>0$ or $Y<0$.

Formally it appears that $B$; are arbitrary constants and on this score

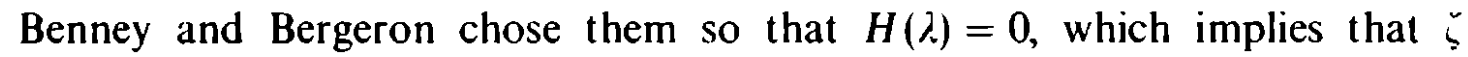
must be discontinuous at the boundary $v=0$ of the cat's eyes. It was thought that those discontinuities are smoothed out, when $i>0$, by thin shear layers of thickness $\lambda^{1 / 2}$ in $Y$, but it was later shown by Brown and Stewardson (1978) that this is impossible. The reader is referred to the original paper for the proof and the result is that $B_{+}=B_{-}=0, K=0$ when $v<0$, and

$$
H(0)=\int_{0}^{\pi}\left\{\frac{1}{2 v^{1 / 2}}-\pi\left[\int_{0}^{2 \pi}\left(v+4 \cos ^{2} \frac{1}{2} x\right)^{1 / 2} d x\right]^{-1}\right\} d v=1.379
$$

the numerical value being due to Smith (1980).

On the other hand, it is also clear that $K(v)$ is an odd function of $Y$ and so the velocity jump $B$ across the critical layer tends to zero as $\lambda \rightarrow 0$. In Figure 10 we display the form of $B$ as a function of $x$ for various values of $\lambda$.

The asymptotic theories of this section may be compared with the numerical studies by Béland $(1976,1978)$ of the evolution of the full solution of the Rossby equation

$$
\frac{\partial}{\partial t} \nabla^{2} \psi-\varepsilon \frac{\partial\left(\psi, \nabla^{2} \psi\right)}{\partial(x, y)}+\frac{\partial \psi}{\partial x}=R^{-1} \nabla^{4} \psi
$$




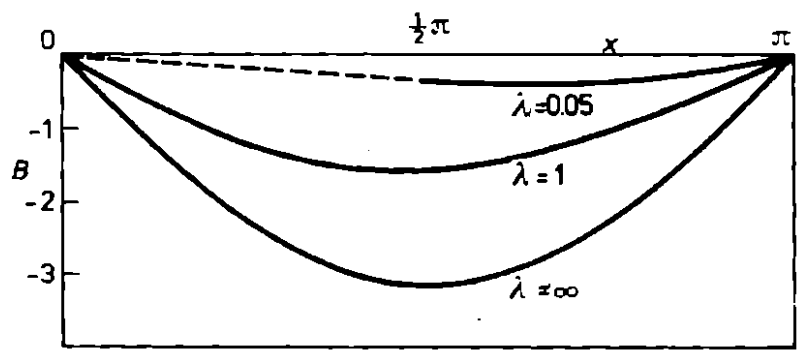

Fig. 10. The velocity jump $B(x, x)$ for various values of $z B(x, x)$ is and odd function of $x$ of period $2 \pi$

with an initial linear profile except near $y=-1.5$, a foreign disturbance $\psi=\cos x$ at $y=2.5$ and a radiation condition at $y=-1.5$. Fifteen harmonics were used in the $x$-direction and finite differences for the integration with respect to $y$ and $t$; the results are internally consistent for $\tau<5$. Bearing in mind the different assumptions of his studies the agreement with the asymptotic theory is remarkable. Thus for $2 \geqslant \lambda \geqslant 0.2$ a steady limit is achieved for the principal harmonic in $B$ and is the same as that found by Haberman (1972). For $\lambda=0$ there are clear signs of the oscillatory behaviour of this harmonic, but as Figure 7 shows, even allowing for an origin shift, there are significant departures from the asymptotic result. It is of interest to note that the scaling laws of the theory hold even up to $\varepsilon=0.2$.

3.4. Summary. We see that when a critical layer is forced by an external oscillating disturbance a velocity jump across the layer of the same shape as the forcing disturbance, but out of phase by $\pi$ is set up in a time $t \sim 1$, but at longer times higher harmonics of the oscillation appear and the non-linear terms tend to make the motion in the critical layer more pathological. This is damped out if some viscosity is present and ultimately the motion becomes steady. However, the viscosity generates a mean vorticity which, although small, is much larger than the original disturbance and gradually spreads right through the fluid. Thus the evolution of the disturbed motion takes to settle down a time comparable to that of the diffusion time of the basic flow and as a result the value of such a steady theory is limited and it must be used with care.

The final form of the velocity jump across the critical layer, as distinct from the jump in mean vorticity, also depends on $\lambda$ and varies from that given by linear theory, when $\lambda \gg 1$, to zero as $\lambda \rightarrow 0$.

\section{Free evolution of critical layers}

4.1. Formulation. Let us now consider the evolution of a shear flow which is initially in a state of marginal stability. Relying on the extended discussion in Sections 2.3 and 2.4, we assume that there is just one normal 
mode, whose wavelength is infinite, and that the associated eigenfunction is analytic everywhere. Hence so long as linear changes only are considered there are no critical layers in marginal stability in the absence of forcing, but as we shall see they arise at second order when non-linear effects are taken into account. The theory which we shall describe here is of general applicability but was worked out (Redekopp (1977); Brown and Stewartson (1979)) in the contex of quasi-geostrophic flow in an atmosphere with a free surface, using Rossby's betaplane model, and we shall do likewise. The governing equation is now

$$
\frac{\partial}{\partial t}\left(\nabla^{2} \boldsymbol{\Psi}-k^{2} \boldsymbol{\Psi}\right)+\frac{\partial\left(\boldsymbol{\Psi}, \nabla^{2} \boldsymbol{\Psi}\right)}{\partial(x, y)}+\beta \frac{\partial \boldsymbol{\Psi}}{\partial x}=R^{-1} \nabla^{4} \boldsymbol{\Psi},
$$

where $k$ is a constant representative of the distorting power of the motion on the free surface and $\beta$ is the (constant) lateral derivative of the Coriolis force. We suppose that there is a mean zonal shear flow $U(y)$ which is in a state of marginal stability. For example

$$
U(y)=\sin y, \quad|y|<\frac{1}{2} \pi
$$

is marginally stable for all $\beta, k$, the phase velocity $c_{0}$ of the mode of infinite wavelength being $-\beta\left(1+k^{2}\right)^{-1}$. Again if $U=\operatorname{sn}(v \mid m),|y|<K(m)$. marginal stability occurs for given values of $m . k$ only at one value of $\beta$. The profile (4.2) will be used as a paradigm when appropriate.

In order to focus attention on the perturbation stream function, we write

$$
\boldsymbol{\Psi}=\int_{0}^{y} U\left(y^{\prime}\right) d y^{\prime}+\psi(\bar{x}, y, t), \quad \bar{x}=x-c_{0} t,
$$

so that the neutral mode is static in our co-ordinate system, where upon

$$
\begin{aligned}
\left(\frac{\partial}{\partial t}+\left(U-c_{0}\right) \frac{\partial}{\partial \bar{x}}+\frac{\partial \psi}{\partial y}\right. & \left.\frac{\partial}{\partial \bar{x}}-\frac{\partial \psi}{\partial \bar{x}} \frac{\partial}{\partial y}\right)\left(\nabla^{2}-k^{2}\right) \psi+ \\
& +\left(\beta+k^{2} U-U^{\prime \prime}\right) \frac{\partial \psi}{\partial x}=R^{-1} \nabla^{4} \psi+R^{-1} \frac{d^{3} U}{d y^{3}} .
\end{aligned}
$$

The overbar on $\bar{x}$ will be omitted henceforth, and $R$ set equal to infinity except when explicitly assumed to be finite.

We have postulated a state of marginal stability, the critical mode having infinite wavelength, and have chosen the axes so that this mode is static to first order. Suppose now that the system is given a small disturbance at time $t=0$, centred at the origin. Then, using also the studies in Section 2.4 on the initial value problem, we may infer that, at large times, $\psi$ takes the form

$$
\psi=A(x, t) \varphi(y)+\psi_{2}(x, y, t)
$$


where $A$ is a slowly-varying function of $x, t, \varphi(y)$ is the eigenfunction of the neutral mode and $\psi_{2}$ is a second order function in some sense. We define the scale of the disturbance by

$$
\varepsilon=\max |A(x, 0)| \ll 1,
$$

and the scales of $x, t$ in $A$ are negative powers of $\varepsilon$. Without specifying what these are, we substitute (4.5) into (4.4) and obtain

$$
\varphi^{\prime \prime}-k^{2} \varphi+\frac{\beta+k^{2} U-U^{\prime \prime}}{U-c_{0}} \varphi=0 ; \quad \varphi\left(y_{1}\right)=\varphi\left(y_{2}\right)=0 .
$$

The equation for $\psi_{2}$ is

$$
\frac{i}{i x}\left\{\left(U-c_{0}\right)\left(\frac{i^{2}}{\partial y^{2}}-k^{2}\right)+\left(\beta+k^{2} U-U^{\prime \prime}\right)\right\} \psi=F(x, y, t),
$$

where

(4.9) $F=\varphi \frac{\partial A \beta+k^{2} U-U^{\prime \prime}}{\partial t}-\varphi^{2} A \frac{\partial A}{\partial x}\left(\frac{\beta+k^{2} U-U^{\prime \prime}}{U-c_{0}}\right)^{\prime}-\varphi \frac{\partial^{3} A}{\partial x^{3}}\left(U-c_{0}\right)$,

and, in general, $\psi_{2}$ has singularity at $y=y_{c}$, where $U=c_{0}$, viz. at the critical level of the flow. This is in contrast with $A(x, t) \varphi(y)$ which satisfies the same equation except that $F$ is zero. In point of fact, since $A \varphi$ is a homogeneous solution of $(4.8), \psi_{2}$ can exist only if $F$ satisfies an integral condition obtained by multiplying both sides by $\varphi /\left(U-c_{0}\right)$ and integrating across the layer. A certain amount of care must be exercised in this operation due to the singularity in $F /\left(U-c_{0}\right)$ and $\psi_{2}$ but the final result can be expressed simply in terms of the velocity jump $B(x, t)$ across the line $U=c_{0}$ defined by

$$
B(x, t)=\underset{y \cdot y_{c}^{+}}{\operatorname{Lt}} \frac{\partial \psi_{2}}{\partial y}-\underset{y \cdot y_{c}^{-}}{\operatorname{Lt}} \frac{\partial \psi_{2}}{\partial y} .
$$

We have

$$
\frac{\partial B}{\partial x}=-\int_{y_{1}}^{y_{2}} \frac{\varphi F d y}{U-c_{0}}
$$

where the asterisk denotes a Cauchy principal value and $\varphi$ has been normalized so that $\varphi\left(y_{c}\right)=1$. Redekopp (1977) has investigated the consequences of (4.11) on the assumption that $B \equiv 0$ in connection with his exploration of the hypothesis that some of the larger scale localized phenomena on Jupiter, such as the Great Red Spot, may arise from solition interactions. We shall see, however, that this hypothesis oversimplifies the problem.

The determination of $B$ depends on a careful study of the nature of the solution in the neighbourhood of $y=y_{c}$. Let us write

$$
\frac{\hat{\imath}^{2} \psi}{\imath y^{2}}=A(x, t)\left[\varphi_{c}^{\prime \prime}+\left(y-y_{c}\right) \varphi_{c}^{\prime \prime \prime}\right]+\zeta(x, y, t)
$$


and assume that the leading term in $\zeta$ is a rapidly varying function of $y$ which is small outside the immediate neighbourhood of $y=y_{c}$. It is convenient at this stage to make a general assumption that $U_{c}^{\prime}\left(y_{c}\right)>0$ (otherwise $\varphi$ may still be singular at $y=y_{c}$ and further care is needed in the special case which arises), to rescale $x, A$ with $\left|U_{c}^{\prime}\left(y_{c}\right)\right|^{-1}$ and to choose $y_{c}=0$. We then find, on substituting (4.12) into (4.4), that

$$
\left(\frac{\partial}{\partial t}+y \frac{\partial}{\partial x}-\frac{\partial A}{\partial x} \frac{\partial}{\partial y}\right) \dot{\zeta}=x \frac{\partial A}{\partial t}+y A \frac{\partial A}{\partial x},
$$

where the constants $\alpha, \hat{\gamma}$ are given by

$$
\alpha=k^{2} U_{c}^{\prime}-U_{c}^{\prime \prime \prime}, \quad \gamma=\frac{1}{2}\left(U_{c}^{\prime \prime \prime}-\frac{U_{c}^{\prime \prime \prime} U_{c}^{\prime \prime}}{U_{c}^{\prime}}\right) .
$$

The velocity jump $B$ is clearly a condition on the integral of $\zeta$ across the critical layer which, for the purpose of solving (4.13), can be supposed to extend from $y=-\infty$ to $y=+\infty$ once we establish (see Section 4.2, below) that it has thickness $O\left(\varepsilon^{1 / 2}\right)$. Finally, we reduce (4.11) to

$$
\frac{\partial}{\partial x} \int_{-\infty}^{\infty} \zeta d y=a \frac{\partial A}{\partial t}-b A \frac{\partial A}{\partial x}+c \frac{\partial^{3} A}{\partial x^{3}},
$$

where

$$
\begin{aligned}
& a=-U_{c}^{\prime 2} \int_{y_{1}}^{y_{2}} \frac{\left(\beta+k^{2} U-U^{\prime \prime}\right) \varphi^{2} d y}{\left(U-c_{0}\right)^{2}}, \\
& b=-U_{c}^{\prime 2} \int_{y_{1}}^{y_{2}}\left(\frac{\beta+k^{2} U-U^{\prime \prime}}{U-c_{0}}\right)^{\prime} \frac{\varphi^{3}}{U-c_{0}} d y, \\
& c=\frac{1}{U_{c}^{\prime}} \int_{y_{1}}^{y_{2}} \varphi^{2} d y .
\end{aligned}
$$

We also need suitable boundary conditions. These are that $\zeta \rightarrow 0$ as $y \rightarrow \pm \infty$ in (4.13) and $\zeta, A$ both $\rightarrow 0$ as $|x| \rightarrow \infty$. The quantities $a, b, c, \alpha, \gamma$ are all constants and provided $|\partial A(x, 0) / \partial x| \ll \varepsilon,(4.13)$ and (4.14) contain all the terms of importance for the evolution of $A$. However, as we shall see, they cannot all be of equal importance for any given large length scale of $A$. Whatever it is, some simplification is possible.

4.2. The linear regime. Suppose now the length scale is initially $O\left(\varepsilon^{-n}\right)$ where $n>0$. We then assume that

$$
t=O\left(\varepsilon^{-m}\right), \quad y=O\left(\varepsilon^{p}\right), \quad \zeta=O\left(\varepsilon^{1+q}\right),
$$


and endeavour 10 lind consistent choices for $m, p, q$ in terms of $n$. It is also necessary to investigate what happens when $\varepsilon^{+m} t$ is large, lest certain terms of (4.13), (4.14) drop out then and are replaced by others. The scaling for $y$ appear to be the same for all $n \geqslant \frac{1}{4}$, namely $p=\frac{1}{2}$, and whenever it is necessary to write down equations in which : does not appear explicitly we shall write

$$
y=\varepsilon^{1 / 2} Y .
$$

For $0<n<4, p=2 n$ but we shall not need this result since the governing equations are then linear.

There are two crucial cases to consider, in one of which $0<n<\frac{1}{4}, q=0$ and $m=3 n$. In this case, which we shall consider here, $A \hat{\imath} A / \partial x$ may be neglected in comparison with $\partial A / \partial t, \hat{S}^{3} A / \hat{\partial} x^{3}$, and (4.13), (4.14) completely solved for $A$. However, as $\varepsilon^{3 / 4} t \rightarrow x$ the behaviour of $A$ is such that $A \partial A / \partial x$ becomes comparable with $\hat{i} A / \hat{\gamma} t$ and non-linear effects are then of significance. In the other case, $n=\frac{1}{2}, m=\frac{3}{2}, q=\frac{1}{2}$, and non-linear terms must be included, but on the other hand $\partial / \hat{s} t$ may be neglected in (4.13): we shall consider this case in Section 4.3, below, and find that there is a shorter time scale which cannot be ignored so that these two cases have much in common.

When $0<n<\frac{1}{4}(4.14)$ reduces to

$$
\left(\frac{\hat{c}}{\partial t}+y \frac{\partial}{\partial x}\right) \dot{\zeta}=x \frac{i A}{\partial t}
$$

and, setting $\zeta=0$ at $t=0$, we have immediately that

$$
\check{\zeta}=x \int_{0}^{1} A_{t}\left(t_{1}, y-t y+x, t_{1}\right) d t_{1}
$$

so that

$$
\frac{\partial}{\partial x} \int_{-x}^{x} \zeta d y=-\alpha \int_{-x}^{*} \frac{A_{t}\left(x^{\prime}, t\right) d x^{\prime}}{x-x^{\prime}}
$$

Now (4.14) becomes

$$
\alpha \int_{x}^{*} \frac{A_{t}\left(x^{\prime}, t\right) d x^{\prime}}{x-x^{\prime}}+a \frac{\partial A}{\partial t}+c \frac{\partial^{3} A}{\partial x^{3}}=0
$$

and, if $\tilde{A}((1), 1)$ is the Fourier transform of $A$ with respect to $x$

$$
\tilde{A}(\omega, t)=\tilde{A}(\omega, 0) \exp \left[\frac{c \omega^{2} t}{a^{2}+\alpha^{2} \pi^{2}}(-\alpha \pi|\omega|+i a \omega)\right] .
$$

Thus if $x c<0$, i.e.. $0<k^{2} U_{c}^{\prime}<U_{c}^{\prime \prime \prime}$, an initial disturbance on a length 
scale $\varepsilon^{-n}$ with $0<n<\frac{1}{4}$ grows unboundedly with $t$ on the time scale $0^{-3 n}$ and the flow must be regarded as unstable to linear disturbances. The theory leading to (4.7) merely established that it is neutrally stable on the time scale $O(1)$. It is far from clear that in this case an initial disturbance could ever take on the form (4.21), due to the problem being badly posed, but in any case a marginally stable flow with $x c<0$ is very hard to construct. Our paradigm (4.2) has $x c>0$ and in such flows (4.21) implies that these initial disturbances decay in magnitude and so are stable but. simultaneously, spread out laterally. Specifically $A \sim \varepsilon^{1-n} t^{-1 / 3}$ over a range of values $\sim t^{1 / 3}$ of $r$ when $s^{3 n} t \gg 1$ and the non-linear term $(\hat{c} A / \partial x)(\partial \zeta / \partial y)$ in $(4.13)$ becomes comparable with $\delta / \partial t$ when $t=O\left(\sigma^{-3 / 4}\right)$ where $\sigma^{3 / 4}=\varepsilon^{1-n}$. At these times $A=O(\sigma)$ and the critical layer thickness $\sim\left(\sigma^{1 / 2}\right)$. The situation is reminiscent of Section 3.2 but it is not now possible to obtain a compete solution even in special circumstances because of the terms on the right-hand sides of (4.13) and (4.14). The best we can do is to set up a series solution in powers of $t$ as in $(3.20)$.

4.3. The solitary wave. Of special interest in the evolutionary process of the disturbance is whether it ultimately may settle down in a similar way to a solitary wave. In that event it may be envisaged as a model describing aspects of fealures of the Jovian atmosphere such as the Great Red Spot. For this phenomenon to occur we must be able to find admissible solutions of the governing equations of the form $f(t-\varepsilon \mu x)$ where $\mu$ is a constant and this implies a non-linear phenomenon. For the non-linear terms in (4.13) and (4.14) to be significant we should think of a length scale $\sim \varepsilon^{-1 / 2}$ and a time scale $\sim \varepsilon^{-3 / 2}$, which is the second crucial case mentioned above and which could arise by evolution from the first. On such a length scale, the governing equation for $\zeta$ reduces 10

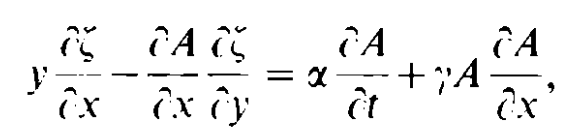

with solution

$$
\zeta= \pm \int_{0}^{x} \frac{x A_{1}\left(x^{\prime}, t\right)+y^{\prime} A\left(x^{\prime}, t\right) A_{x^{\prime}}\left(x^{\prime}, t\right)}{y^{2}+2 A(x, t)-2 A\left(x^{\prime}, t\right)} d x^{\prime}
$$

according as $y>0$ or $y<0$, on any curve on which

$$
u=y^{2}+2 A(x, t)
$$

is constant. Thus, if the curve $u=$ constant extends to $x=-x$ which it must do if $y^{2}$ is large enough. the lower limit of integration can be taken as $-x$. From a detailed study of this equation Brown and Stewartson (1979) 
infer that the functions $A$ must satisfy certain restrictive conditions. One class of function which is acceptable satisfies the equation $A_{x}=g(A) A_{t}$ provided $A \leqslant 0$ and has a single minimum. For example, if $A_{t}=-\varepsilon \mu A_{x}$

$$
\zeta=\varepsilon \alpha \mu\left(y \mp\left(y^{2}+2 A\right)^{1 / 2}\right)-\frac{1}{3} \gamma\left\{y^{3} \mp\left(y^{2}+2 A\right)^{3 / 2}+3 A y\right\}
$$

if $u \equiv y^{2}+2 A>0$, and

$$
\zeta=\varepsilon \alpha \mu y-\gamma\left(\frac{1}{3} y^{3}+A y\right)+F(u),
$$

if $u<0$, where $F$ is a function to be found, except that presumably $F(0)=0$ to ensure continuity of $\zeta$. An important requirement for this solution is that the lines on which $u=$ constant are either closed and cross the line $y=0$, or extend from $x=-\infty$ to $x=+\infty$. If $A>0$ then there are lines, on which $u=$ constant, which cross the line $y=0$ and extend to $x= \pm \infty$, where, of necessity, contradictory requirements are placed on $F(u)$, the homogeneous solution of (4.22). Granted that $A_{t}=-\varepsilon \mu A_{x}, A<0$ and taking $F=0$ we obtain a solitary wave for $A$ namely

$$
\hat{A}_{0}(x, t)=-\frac{12 \varepsilon c}{b} \operatorname{sech}^{2} \varepsilon^{1 / 2}\left(x-\frac{4 \varepsilon c t}{a}\right) .
$$

The validity of (4.27) appears to depend on $\zeta$ being quasi-steady, i.e., that $\partial \zeta / \hat{t} t$ is negligible in comparison with the terms retained in (4.22), and this is not the case. Nevertheless, we may show, by a parallel argument to that in Section 3.2, that it is consistent to assume the velocity jump $B$ across the critical layer is zero. In order to do this, we replace $A$ in (4.13) by its quasisteady form (4.27) and, for simplicity, set $\gamma=0$. This equation may now easily be solved for $\zeta$ given that $\zeta=0$ at $t=0$ and we find, as in Section 3.2, that inside the lines $y^{2}+2 \hat{A}=0, \zeta$ oscillates finitely whereas, outside, it tends to a limit as $s . t \rightarrow \infty$. The left-hand side of (4.14) behaves like $t^{-1 / 2}$ when $\varepsilon t$ is large as required.

Previously this oscillation was removed by the introduction of a weak viscosity (Section 3.3) but there and in the present instance such a generalization leads to difficulty since the basic flow does not now satisfy the full Navier-Stokes equations. The consequences in the earlier work were not serious since the time-scale of the evolution of the mean shear is much larger then that of the oscillatory flow in the critical layer and so this formal contradiction could be ignored.

Now the disturbance is effectively to the mean shear and so the evolution of the two must be considered together. The equation for $\zeta,(4.22)$, changes to

$$
y \frac{\partial \zeta}{\partial x}-\frac{\partial A}{\partial x} \frac{\partial \zeta}{\partial y}-R^{-1} \frac{\partial^{2} \zeta}{\partial y^{2}}=\alpha \frac{\partial A}{\partial t}+\gamma A \frac{\partial A}{\partial x}-R^{-1} U_{c}^{\prime \prime \prime} .
$$

We know that when $R$ is large enough $\zeta=O\left(\varepsilon^{3 / 2}\right)$ and $y=O\left(\varepsilon^{1 / 2}\right)$, and so 
the forcing from the diffusion of the basic shear dominates the smoothing effect of viscosity on the left-hand side of (4.28). Further, as soon as this term matters the notion that $\zeta \rightarrow 0$ as $x \rightarrow \pm \infty$ is lost. Similar remarks may be made for any value of $\bar{\lambda}_{.}=\left(R c^{2}\right)^{-1}>0$. It is only worthwhile considering viscous effects if

$$
U_{c}^{\prime \prime \prime}=0
$$

there is, incidentally, another requirement on $U$ needed to prevent a contribution to the right-hand side of (4.14) from the mean shear. Brown and Stewartson (1979) attempted to determine $F$ when both these requirements are met by using an extension of the Benney-Bergeron theory but they overlooked an additional difficulty. For if we write

$$
\zeta=\zeta_{0}(x, y)+R^{-1} \check{\zeta}_{1}(x, y)+O\left(R^{-2}\right)
$$

where $\zeta_{0}$ is given by (4.25) when $u>0$ and (4.26) when $u<0$, we find that it is impossible for $\zeta_{1}$ to tend to zero as $|x| \rightarrow \infty$ in the region where $u>0$.

4.4. Summary. We have shown that the evolution of an initial disturbance to a marginally stable shear flow depends on the scale over which it is made. From the discussion in Section 2.4 we may expect that to begin with there is a sorting out process controlled entirely by the linear equations, at the end of which most components of the disturbance are decaying algebraically with time leaving only a mode with a large wavelength. If its scale is not too long $\left(<\delta^{-1 / 4}\right)$ where $\delta$ is a representative magnitude of the stream function at the time being considered, then an examination of the stability characteristics on a longer time-scale shows that the basic flow may be either stable or unstable although the second possibility is difficult to realize. If stable the disturbance decays and spreads outwards until eventually its length-scale reaches $\delta^{-1 / 4}$, whereupon non-linear effects become important. For length scales $\geqslant \delta^{-1 / 4}$ we have been able to show that it is possible for a solitary wave to be set up, similar to that occurring in the Korteweg de Vries equation. Associated with this wave is a weak finite oscillation in the critical layer of the disturbance. It does not seem possible to generalize the solitary wave to include soliton interactions because of the special properties which must be satisfied in the critical layer and the oscillation can only be removed by the action of viscosity but this destroys the basis of the solitary wave concept $(\zeta \rightarrow 0$ as $|x| \rightarrow x)$.

\section{Stratified flows}

5.1. Introduction. Critical layers are also commonly found in the study of unstable parallel horizontal shear flows when in addition the density of the fluid varies under the action of gravity, such situations occurring frequently in the atmosphere and oceans. The fluid motion is described by 
equations deduced from the full equations of motion after making the Oberbeck. Boussinesq approximation in which density variations are neglected except for the buoyancy term of the vertical component of the momentum equation. Mihaljan (1962) has shown that if the equation of state gives the density as a linear function of temperaturc only, and if the density variations are small and we take the reduced shear velocity and density change in the basic flow to be $U(y), R(y)$ respectively, then on disturbing the flow we find that the perturbations $\varepsilon \psi(x, y, t), \varepsilon T(x, y, t)$ in the stream function and the temperature satisfy

$$
\left(\frac{\hat{c}}{\partial t}+U(y) \frac{\partial}{\partial x}\right) \nabla^{2} \psi-U^{\prime \prime}(y) \frac{\partial \psi}{\partial x}-\frac{\partial\left(\psi, V^{2} \psi\right)}{\partial(x, y)}+R i \frac{\partial T}{\partial x}=0
$$

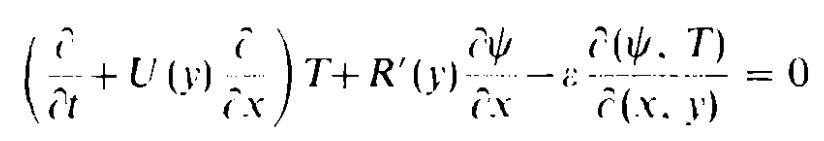

neglecting, for the time being, the effects of viscosity. Here $s$ is a number, formally arbitrary, but which later on we shall take to be very small, and $R i>0$ is the overall Richardson number, proportional to the coefficient of the buoyancy force divided by the square of the velocity difference across the layer. Thus $R^{\prime}<0$ corresponds 10 a stably stratified atmosphere and, as we shall see, so long as $\left[-R^{\prime}\left(U^{\prime}\right)^{2}\right] R i \geqslant \frac{1}{4}$ everywhere the shear flow is stable irrespective of the form of $U(y)$. If $R i=0$, equations (5.1) reduce to the equations for an unstratified fluid and the form of $U(v)$ is of crucial importance to the stability problem. For boundary conditions we take

$$
\psi=0 \text { at } \quad y=y_{1}, y_{2}
$$

in cases where the fluid is confined within rigid horizontal boundaries, and allow $y_{1}$ and/or $y_{2}$ to tend to infinity when the flows are semi-bounded or unbounded. In addition we shall suppose that $\psi, T$ are prescribed at $t=0$ and are either periodic in $x$ or are centred at $x=0$, vanishing as $|x| \rightarrow x$. As in the theory of unstratified flows we assume throughout that $U(y), R(y)$ are smooth functions of $y$ and specifically exclude any discussion of basic profiles with discontinuities or even corners.

5.2. Linearized theory: the Taylor-Goldstein-Haurwitz (TGH) equation. When $\varepsilon \ll 1$ the non-linear terms of (5.1) may be neglected and the equations reduce to

$$
\left(\frac{\partial}{\partial t}+U \frac{\hat{\partial}}{\partial x}\right)^{2} \nabla^{2} \psi-U^{\prime \prime}(y)\left(\frac{\partial}{\partial t}+U \frac{\partial}{\partial x}\right) \frac{\partial \psi}{\partial x}-R i R^{\prime}(y) \frac{\partial^{2} \psi}{\partial x^{2}}=0 .
$$

It is now appropriate to write

$$
\psi=e^{i z(x-c t)} \varphi(y)
$$


whereupon $\varphi$ satisfies

$$
(U-c)\left(\varphi^{\prime \prime}-x^{2} \varphi\right)-U^{\prime \prime} \varphi-\frac{\operatorname{Ri} R^{\prime}\left(y^{\prime}\right) \varphi}{U-c}=0
$$

to which we shall refer as the TGH equation acknowledging its simultaneous and independent discovery by Taylor, Goldstein and Haurwitz (1931). The function $-R^{\prime}(y)$ is often set equal to $N^{2}(y), N$ being known as the BruntVãisãlã frequency. Since $\varphi=0$ at $y=y_{1}, y_{2}$ the equation has non-trivial solutions only for a set of values of $c$, which we expect to be discrete. Further, since the complex conjugates $\tilde{\varphi}$ and $\tilde{c}$ of $\varphi$ and $c$ are also solutions of (5.5), if $c_{i} \neq 0$ for any admissible $c$, the basic flow is unstable since there exists a weak initial disturbance that ultimately grows without limit.

In our survey of the properties of the TGH equation we first observe that, although Rayleigh's equation, to which (5.5) reduces when $R i=0$, has no solution in which $c_{i}=0$ and $c_{r}$ is outside the range of $U$ the same is not true when $R i>0$ and $-R^{\prime}(y)>0$. Details of the properties of such solutions may be found in Banks, Drazin and Zaturska (1976) and the literature quoted therein, but the existence of such waves may bc expected from the following two examples with $R i \gg 1$. Take $-R^{\prime}(y)=N^{2}=1, y_{1}=-\pi$, $y_{2}=\pi$ and then

$$
c \sim \pm\left(x^{2}+\frac{1}{4} n^{2}\right)^{1 / 2} R i^{1 / 2}, \quad \varphi \sim \sin \frac{1}{2} n(\pi+y), \quad n=1,2,3, \ldots
$$

Again, take $N^{2}=\operatorname{sech}^{2} y, y_{1}=-\propto, y_{2}=\propto$ and then

$$
c \sim \pm \frac{2 R i^{1 / 2}}{\left((2 n-1+2 x)^{2}-1\right)^{1 / 2}}, \quad \varphi \sim \operatorname{sech}^{\gamma} y \stackrel{C}{C=1}^{x+1 / 2}(\tanh y)
$$

C being a Gegenbauer polynomial (Groen (1948)). In each of these examples Banks at al. show, for a particular shear flow $U(y)$, that like solutions may be found for all $R i \geqslant 0$ and that $c \rightarrow(\operatorname{Max} U)_{+}$or $c \rightarrow(\operatorname{Min} U)_{-}$as $R i \rightarrow 0_{+}$. The eigensolutions associated with these values of $c$ do not seem to lead to any instabilities; since they do not possess critical layers their properties are outside the scope of this review and we shall not discuss them further.

Let us now examine the modes for which $\operatorname{Min} U<c_{r}<\operatorname{Max} U$ so that if $c_{i} \neq 0$ they include a critical layer. Their main properties are given by two theorems. The first is due to Howard (1961) and is known as the semicircle theorem. It asserts that if $c_{i} \neq 0$ then

$$
\left[c_{r}-\frac{1}{2}(\operatorname{Max} U+\operatorname{Min} U)\right]^{2}+c_{i}^{2}=\left[\frac{1}{2}(\operatorname{Max} U-\operatorname{Min} U)\right]^{2}
$$

and gives bounds on the possible values of $c$. The second theorem is due to Miles (1961) and Howard (1961) and asserts that a necessary condition for $c_{i} \neq 0$ is that

$$
J(y) \equiv \frac{-R i R^{\prime}(y)}{\left[U^{\prime}(y)\right]^{2}}<\frac{1}{4}
$$


somewhere in the field of flow. This condition may also be suffucient. Thus if $U(y)=-R(y)=\tanh y^{\prime}, y_{1}=-x, y_{2}=+\infty$ and $R i=\frac{1}{4}$ then $J(y) \geqslant \frac{1}{4}$ and a neutral mode with $\alpha<\frac{1}{2}$ can be found; if $R i<\frac{1}{4}$ unstable modes have been computed (Hazell (1972)).

For neutral modes with $c_{\mathrm{i}}=0$ the eigensolution has a singularity at $y=y_{c}$ and nearby $\varphi$ has the form

$$
A_{ \pm}\left|y-y_{c}\right|^{1 / 2 \cdot \overline{1 / 4}-J_{c}^{-}}+B_{ \pm}\left|y-y_{c}\right|^{1 / 2+\sqrt{1 / 4-J_{c}}}, \quad J_{c}=J\left(y_{c}\right)
$$

where $A_{+}, B_{+}$are constants whose ratio is determined by the overall properties of the solution in $y>y_{c}$ or $y<y_{c}$. As with the Rayleigh equation (Section 2.2) there are three ways by which the critical layer at $y=y_{c}$ may be crossed. The lirst is to adapt the Benney-Bergeron theory of critical layers, taking account of viscosity and non-lineary there, to bridge the gap between the two solutions. This procedure has been examined by Kelly and Maslowe (1970) and Maslowe (1972), but the difficulty of dealing with the mean shear induced by the critical layer has prevented a complete study from being carried out.

The second is to appeal to analytical continuation, or alternately to take $c_{i}>0$ and let $c_{i} \rightarrow 0$, which leads to the conclusion that a solution is only possible if $A$ or $B$ is zero on both sides. This cannot be achieved unless $J_{c} \leqslant \frac{1}{4}$ since $A$ and $B$ are complex conjugates for $J_{c}>\frac{1}{4}$ and we conclude (Miles (1961)) that there are no modes of this type if $J(y)>\frac{1}{4}$. The internal waves are present of course but they do not have critical layers. For $J_{c}<\frac{1}{4}$ it might be argued that we can choose $A B=0$ for some $c$ and the existence of neutral modes would seem to confirm this. The study of the neutral modes of (5.5) and of its analogue for rotating systems (see Pedley (1968); Maslowe (1974) for a derivation of the relevant equation and an account of its properties) must, however, be regarded as incomplete at the present time.

The third method is to consider an initial value problem and, in particular, to look at the growth of the stream function $\psi$ as a function of time in the neighbourhood of $y=y_{c}$. As with the method of analytic continuation this shows that the two solutions (5.10) may only be joined by a finite but growing critical layer at large values of $t$ if $A_{+} / B_{+}$and $A_{-} / B_{-}$are in a certain proportion - one which its is impossible to achieve if $J_{c}>\frac{1}{4}$.

The conclusion is that, just as in the Rayleigh equation, free oscillations with critical layers cannot occur when the flow is stable although we now can have free oscillations without critical layers. Critical layers can, however, occur if the oscillation is forced, for example by prescribing $\varphi, \alpha$ and $c$ at $y=y^{*}$ where $y^{*}>y_{c}$. The linear properties of the critical layer in such problems were first studied by Booker and Bretherton (1967) and by many authors since. They showed that the critical layer has a very special property, which they termed absorption, of decreasing the amplitude of the oncoming wave by a factor $O\left(e^{-2 v \pi}\right)$ where $v=\left(J_{c}-\frac{1}{4}\right)^{1 / 2}$, thus reducing it to a 
vanishing small value once $J_{c}$ is appreciably greater than $\frac{1}{4}$. For example, if $J_{c}=0.3, e^{-2 v \pi} \simeq \frac{1}{4}$ and if $J_{c}=0.5, e^{-2 v \pi}=1 / 25$. Moreover the reflected wave is also very weak. Later we shall discuss this phenomenon in more detail paying especial attention to non-linear aspects. It is worth noting here that in more complicated flows, e.g., with rotation or in the presence of magnetic fields, a valve effect can also occur with one type of wave passing smoothly through the critical layer while another is absorbed (Acheson (1973)).

One other class of solution to the linearized equation (5.3) may be identified. As with Rayleigh's equation (2.4) we may consider the continuous spectrum which arises from the branch points of the Laplace transform of the solution with respect to $t$. Its form may be written down directly from (5.3) on the assumption that, when $t \geqslant 1$,

$$
\psi \sim e^{i x[x-U(y)) !} t^{-3 / 2 \pm i v(y)}\left[H(y)+O\left(\frac{\log t}{t}\right)\right]
$$

where $v(y), H(y)$ are functions of $y$ to be found. It follows immediately that

$$
v^{2}(y)=-\frac{1}{4}-\frac{R^{\prime}}{U^{\prime 2}} R i
$$

that $H(y)$ is arbitrary and that the expression in brackets may be regarded as a series in descending powers of $t$ and ascending powers of $\log t$ whose coefficients are functions of $y$. This form was first written down by Eliassen Höiland, and Riis (1953) and subsequently by Booker and Bretherton (1967) for a uniform shear. There has, however, been a long-standing controversy about its correctness which has recently been resolved by Brown and Stewartson (1980) who also show how the unknown functions may be found in an initial value problem in terms of the values of $\psi$ and $\hat{\psi} \psi / \hat{c} t$ at $t=0$. In such problems the final state of $\psi$ is a linear combination of solutions of the TGH equation and functions such as (5.11). These modes can occur whether $v$ is positive or negative, but are of greatest importance when $\operatorname{Min} J>\frac{1}{4}$ so that no unstable wave-like modes can occur. For example, these modes are important in the absorption process of incident waves at large values of $J$ discussed earlier. For such problems the function $H(y)$ develops a singularity at the critical layer and it turns out that there is a thin transition layer when $t \gg 1$ located at $y t=O\left(J^{1 / 2}\right)$ across which the incident wave is converted into one of these modes (Brown and Stewartson (1981)). Thus energy which had hitherto been travelling across the shear layer now travels parallel to it with the local fluid velocity. This is the physical significance of the concept of critical layer absorption.

5.3. Non-linear evolution for $R i=\frac{1}{4}$. We now consider the non-linear evolution of a small disturbance imposed at $t=0$ on a stratified fluid in 
shear flow when it is marginally unstable. In order to fix the problem we take

$$
R^{\prime}(y)=-\operatorname{sech}^{2} y, \quad U(y)=\tanh y, \quad J(y)=R i \cos ^{2} y .
$$

If $R i<\frac{1}{4}$ this flow is unstable and we may expect that any initial disturbance rapidly becomes too complicated to follow analytically, while if $R i>\frac{1}{4}$ there are no dominant modes whose amplitude variation can be traced. The one case which we can discuss is when $R i=\frac{1}{4}-$, so that the flow is marginally unstable and the dominant wave has

$$
\alpha=\frac{1}{2}, \quad c=0, \quad \varphi=a_{+}(\operatorname{sech} y)^{1 / 2}\left(\frac{1}{2} \tanh |y|\right)^{1 / 2}
$$

according as $y>0$ or $y<0$ with

$$
a_{ \pm} \propto e^{i(1-4 R i) / 4}
$$

(Miles (1963); Brown, Rosen and Maslowe (1981)). This form of solution is singular at $y=0$ which is the critical level and must be replaced by another which is regular at $y=0$ but has a more complicated dependence on $t$, namely

$$
\psi=-e^{i x / 2} \frac{A}{2 \pi^{1 / 2}} \int_{0}^{t} \frac{e^{-i y u / 2}}{u^{3 / 2}} d u+\text { c.c. }
$$

where the ${ }^{*}$ denotes that the finite part is to be taken and c.c. means complex conjugate. Further, matching (5.15) with (5.13) shows that

$$
A e^{ \pm \pi i / 4}=a_{ \pm}, \quad \text { i.e., } \quad a_{-}=i a_{+},
$$

confirming the identity of conclusions to be drawn, from an initial value study and from insistence on analyticity, about the relationship between the solution in $y>y_{c}$, and in $y<y_{c}$. This form of the dominant mode is discussed by Brown and Stewartson (1978) whose argument we follow here but they omitted the precise form of the amplitude dependence (5.14) of $a_{ \pm}$ on $t$. This may also be deduced from Howard's theory (1963) of the behaviour of $c$ near the stability boundary.

The explicit form obtained for $\psi$ in (5.15) implies that the horizontal velocity and the temperature become large when $t$ is large, even when $R i$ $=\frac{1}{4}-0$. Thus at $y=0,(5.15)$ implies that

$$
T=\frac{i A}{\pi^{1 / 2}} e^{i x / 2} t^{1 / 2}+\text { c.c. }
$$

so linear theory must eventually fail. This failure is largely confined to the critical layer in the next stage of evolution of the disturbance and can be 
analysed separately from the remainer of the flow field by setting $R i=\frac{1}{4}$ for the present and rescaling the variables. We write

$$
\begin{gathered}
y=\varepsilon^{2 / 3} Y, \quad \tau=\varepsilon^{2 / 3} t, \quad R i=\frac{1}{4}, \\
\psi=\varepsilon^{1 / 3} \Psi(x, Y, \tau), \quad T=\varepsilon^{-1 / 3}\left\{2 \frac{\partial \Psi}{\partial Y}+\frac{\partial \Phi}{\partial Y}\right\},
\end{gathered}
$$

whereupon

$$
\begin{aligned}
& \left(\frac{\partial}{\partial \tau}+Y \frac{\partial}{\partial x}\right) \frac{\partial \Phi}{\partial Y}-\frac{1 \partial \Phi}{2 \partial x}=-\left(\frac{\partial \Psi}{\partial Y Y} \frac{\partial^{2} \Phi}{\partial x \partial Y}-\frac{\partial \Psi}{\partial x} \frac{\partial^{2} \Phi}{\partial Y^{2}}\right) \text {. } \\
& \left(\frac{\partial}{\partial \tau}+Y \frac{\partial}{\partial x}\right) \frac{\partial \Psi}{\partial Y}-\frac{1}{2} \frac{\partial \Psi}{\partial x}=-\frac{1}{4} \frac{\partial \Phi}{\partial x}-\left(\frac{\partial \Psi}{\partial Y} \frac{\partial^{2} \Psi}{\partial x \partial Y}-\frac{\partial \Psi}{\partial x} \frac{\partial^{2} \Psi}{\partial Y^{2}}\right)
\end{aligned}
$$

together with initial conditions

$$
\Psi=e^{i x / 2} \Psi_{11}(Y, \tau)+\text { c.c }, \quad \Phi=0,
$$

$$
\psi_{11}=-\frac{A}{2 \pi^{1 / 2}} \int_{0}^{*} \frac{e^{-i Y u / 2}}{u^{3 / 2}} d u .
$$

Before discussing the solution of these equations some preliminary comments about the scaling laws of (5.18) may be useful, since they are not quite in their final form. As stated, the solution of (5.19) is formally independent of $\varepsilon$ and may be set up as a series in ascending powers of $\tau$ whose coefficients are functions of $\gamma_{\tau}$ and $x$. In addition, the various terms can be computed without reference to the solution outside the critical layer although this means that the solution contains undetermined constants. These may be found by reference to the solution outside but the matching procedure is not quite as straightforward as is normally the case and necessitates a rescaling of the variables in the critical layer. It may be done either by assuming that $A$ is not $O(1)$ but actually $O\left(\varepsilon^{1 / 6}\right)$ or that the time scale in $t$ is not $O\left(\varepsilon^{-2 / 3}\right)$ but $O\left(s^{-4 / 7}\right)$. In the interest of a purely formal expansion procedure therefore there is something to be said for introducing these scales right away, but since the real reason for the choice only becomes apparent after a long calculation, and there is no saving in computation time if the correct scales are adopted, we shall stick to these more natural scales and make any adjustments needed at the appriopriate stage in the argument.

A basic feature of the solution in the critical layer is its form as $|y| \rightarrow \infty$. From a study of the equations obtained from (5.19) by neglecting the right-hand side. we find that

$$
\Psi-e^{ \pm: i / 4}\left|\frac{1}{2} Y\right|^{1 / 2}\left(-\frac{1}{4} F\left|\frac{1}{2} Y\right| \pm \frac{1}{2} \pi i\right)+E+\text { c.c. }
$$

$$
\Phi \sim\left|\frac{1}{2} Y\right|^{1 / 2} F e^{ \pm \pi i / 4}+\text { c.c. }
$$


as $Y \rightarrow \pm \propto$ where $E, F$ are arbitrary functions of $(x, \tau)$ to be found. Moreover, we know from the initial conditions (5.20), that

$$
E \rightarrow A e^{i x / 2}, \quad F \rightarrow 0 \quad \text { as } \tau \rightarrow 0 .
$$

The expansion of the solution in powers of $\tau$ proceeds by successive substitution into the right-hand side of (5.19) and so we may anticipate that in general $F \neq 0$ and what is more to the point, that $F$ takes on different values as $Y \rightarrow \pm \infty$. Both $E$ and $F$ also contain arbitrary functions. Hence we can write the asymptotic form for $\Psi$ in the full solution as

$$
\begin{aligned}
\Psi \sim e^{-\pi / 4}\left|\frac{1}{2} Y\right|^{1 / 2}\left[E-\frac{1}{4} F\left(\log \left|\frac{1}{2} Y\right|-\frac{1}{2} \pi i\right)\right]+\text { c.c., } \quad \text { as } Y \cdots-\infty, \\
\Psi \sim e^{\pi i / 4}\left(\frac{1}{2} Y\right)^{1 / 2}\left[E-\frac{1}{4} F\left(\log \frac{1}{2} Y+\frac{1}{2} \pi i\right)\right]+ \\
+I-\frac{1}{4} J\left(\log \frac{1}{2} Y+\frac{1}{2} \pi i\right)+\text { c.c., } \quad \text { as } Y \rightarrow+\infty,
\end{aligned}
$$

where $E$ and $F$ are again arbitrary and at any stage of the series expansion $I$ and $J$ can be found in terms of $E$ and $F$.

The leading terms in the expansions of $I$ and $J$ have been computed on the assumption that $E$ and $F$ are forced by $A$ only. Other forcing functions may be considered but it is this contribution only that we need to deduce the leading terms in the evolution equation for the initial disturbance, We have

$$
\begin{aligned}
& I=\frac{2}{9} i A|A|^{2}(14+\pi)\left(\frac{1}{8} \tau\right)^{3} e^{i x / 2}+3.37 i A|A|^{2}\left(\frac{1}{8} \tau\right)^{9 / 2} e^{i x}+O\left(\tau^{6}\right), \\
& J=-4.04 i A|A|^{4}\left(\frac{1}{8} \tau\right)^{6} e^{i x / 2}+i \beta_{3} A|A|^{4} e^{3 i x / 2}\left(\frac{1}{8} \tau\right)^{6}+O\left(\tau^{15 / 2}\right),
\end{aligned}
$$

where $\beta_{3}$ is a constant not yet computed.

In the matching problem with the solution outside the critical layer the first term to constrain the value of $A$ is the leading coefficient in (5.26), since all the others explicitly quoted can be accommodated by a suitable choice of a homogeneous external solution or choice of $E$ and $F$. The presence of a non-zero coefficient of $e^{i x / 2}$, with a logarithmic factor. contradicts the requirements of a solution of (5.5) with $\alpha=\frac{1}{2}$ for then this factor must be explicitly absent. A match is possible only if we allow $A$ to vary with $\tau$ in which case a forcing term proportional to $\hat{C} A / \hat{\tau} \tau$ appears on the right-hand side of (5.5) and leads to a logarithmic term in $y$. A match of this term with (5.23) and (5.24) is now possible leading to an equation for $A$. At this stage we may also allow for the fact that $R i$ is not quite equal to $\frac{1}{4}$ which also gives a forcing term to (5.5), and finally the matching process leads to

$$
\frac{d A}{d \tau}=\varepsilon^{-2 / 3}\left(\frac{1}{4}-R i\right) A+\frac{1}{8}\left(\frac{\tau}{8}\right)^{6} \beta_{1} \varepsilon^{-2 / 3}|A|^{4} A,
$$

where $\beta_{1}$ was computed to be equal to 0.367 and $\frac{1}{4}-R i$ is small and positive. If we revert to the unscaled temporal variable $t$, see (5.18), (5.27) becomes

$$
\frac{d A^{*}}{d t}=\left(\frac{1}{4}-R i\right) A^{*}+\frac{1}{8}\left(\frac{t}{8}\right)^{6} \beta_{1}\left|A^{*}\right|^{4} A^{*},
$$


where $\varepsilon A=A^{*}$ so that $A^{*}$ is also the unscaled amplitude of the disturbance. We now see that the non-linear evolution of an initial weak disturbance when $\frac{1}{4}-R i$ is small and positive takes place over a time scale $\sim\left(\frac{1}{4}-R i\right)^{-1}$ when its amplitude $\sim\left(\frac{1}{4}-R i\right)^{-7 / 4}$. In terms of $\varepsilon$ taken to be a representative amplitude of the disturbance, the non-linear evolutionary time scale is $O\left(\varepsilon^{2 / 21}\right)$ in $\tau$ or $O\left(\varepsilon^{-4 / 7}\right)$ in $t$, as mentioned earlier.

The reduced' form of evolutionary equation

$$
\frac{d B}{d t_{1}}=B+t_{1}^{6}|B|^{4} B
$$

derived from (5.28) by an obvious scaling, is similar to the Landau-Stuart equation

$$
\frac{d C}{d t_{1}}=C+k|C|^{2} C \quad(k=\text { constant }),
$$

of classical non-linear stability theory (Stuart (1960)). We may conclude that, just as with (5.30), $B \rightarrow \infty$ at a finite value of $t$ and hence that the basic flow is supercritically unstable. We may not conclude that it is subcritically unstable because the linear theory corresponding to (5.13) and (5.14) does not exist when $R i>\frac{1}{4}$. For $R i=\frac{1}{4}+0$ there are no modal solutions of the form (5.4) which include a critical layer. The only solutions which exist in this form have $|c|>1$, correspond to internal waves, and in particular to not have the non-linear evolution of the form we have been discussing here. Should three-dimensional disturbances be permitted then reasonant triads or tetrads may occur (McEwan, Mander and Smith, (1972)) but the instability is bounded in amplitude. The parametric dependence of the non-linear disturbances in $R i$ in the neighbourhood of $R i=\frac{1}{4}$ is certainly peculiar and has by no means been fully resolved. The presence of the factor $t_{1}^{6}$ in (5.29) is worth noting: it may be associated with the fact that the basic linear solution (5.13) is not smooth everywhere and so the standard integrals of Stuart-Landau theory do not converge. The power of $t$ thus signifies that the integrals are evaluated at a large but finite time. Finally, we draw attention to the high power of $B$ on the right-hand side, an unusual feature of these theories (but see Roberts and Stewartson (1974)).

A study on the lines of the preceding section when the effect of viscosity is comparable with that of non-linearity has been carried out by Brown, Rosen and Maslowe (1981). The appropriate parameter is $\lambda_{1}$ where $\lambda_{1}$ $=\left(R \varepsilon^{2}\right)^{-1}$ is the stratified equivalent of the parameter $\lambda$ defined in (3.14), and this is taken to be large so that the investigation is analogous to that of Tietjens rather than that of Benney and Bergeron. The time scale of interest has

$$
R^{1 / 3} \ll t \ll R,
$$

the implication of these inequalities being that the time is sufficiently long for 
the critical layer to have become quasi-steady but not long enough for the mean llow, which does not satisfy the Navier-Stokes equations, to diffuse on this account. This difficulty, inherent in viscous stability theory, is noted in Section 2.2. There is a distortion of the mean flow of the Haberman type similar to that of Section 3.3 for the homogeneous situation and the effect of this is included.

The equations for the viscous problem are (5.1) with the zeroes on the right-hand sides replaced by $R^{-1} \nabla^{4} \psi$ and $(R \mathrm{Pr})^{-1} \Gamma^{2} T$, respectively. Here Pr is the Prandil number and is set equal to unity in the analytical investigation but not necessarily in the numerical work which is an extension of the weakly non-linear theory of Maslowe (1977). The terms in $U^{\prime \prime \prime}(y)$, $R^{\prime \prime}(y)$ are neglected as justified above. The basic profiles are exactly as in (5.12) and the marginally unstable mode whose development is examined is again that in (5.13). In the neighbourhood of the critical layer at $y=0$ the solution for the stream function is, when $\operatorname{Pr}=1$ (5.15) with a factor $\exp \left[-u^{3} /(12 R)\right]$ in the numerator of the integrand, and on a time-scale on which the critical layer is quasi-steady, i.e., only $A$ is a function of time, the upper limit $t$ tends to infinity. When $\operatorname{Pr} \neq 1$ no such simple solution for $\psi$ is available. Again the non-linear terms become important in the critical layer the thickness of which is now $O\left(R^{-1 / 3}\right)$. The appropriate variables in the region are, when $\operatorname{Pr}=1$, of the form (5.18) but with

$$
\begin{aligned}
y=R^{-1 / 3} Y, \quad \tau & =R^{-1 / 3} t, \quad \psi=R^{-1 / 6} \Psi(x, Y, \tau), \\
T & =R^{1 / 6}\left(2 \frac{\partial \Psi}{\partial Y}+\frac{\partial \Phi}{\partial Y}\right),
\end{aligned}
$$

and the resulting equations are (5.18) and (5.19) with additional terms $\hat{\imath}^{3} \Phi / \hat{\partial} Y^{3}, \hat{\imath}^{3} \Psi / \hat{\partial} Y^{3}$ on the right-hand sides and the non-linear terms multiplied by $\lambda_{1}^{-1 / 2}$. A solution is sought in powers of $\lambda_{1}^{-1 / 2}$ which is analogous to the expansion in powers of $\tau$ in Section 5.4. The terms in $\hat{c} / \hat{\tau} \tau$ in the equations may be set equal to zero when $t \gg R^{1 / 3}$ but are retained to order $\lambda_{1}^{-1 / 2}$ to illustrate the diffusive effect of the $x$-independent term that results in a distortion of the mean flow of order $\varepsilon^{2} R^{2 / 3}$.

Again as in Section 5.4 there is eventually a restriction on the function $A$ as the outer flow is unable to accept a leading-harmonic logarithmic contribution forced on it by the critical layer unless $A$ depends on $t$. This amplitude equation is of the form

$$
\frac{d A^{*}}{d t}=\left(\frac{1}{4}-R i\right) A^{*}+a_{2}^{*}\left|A^{*}\right|^{2} A^{*}+a_{4}^{*}\left|A^{*}\right|^{4} A^{*},
$$

where $a_{2}^{*}$ and $a_{4}^{*}$ are functions of $R$ and $\operatorname{Pr}$ with $R i=\frac{1}{4}$. Strictly, the term involving $a_{4}^{*}$ is relevant only if $a_{2}^{*}=O\left(R^{1 / 3}\right)$. If $\operatorname{Pr} \neq 1, a_{2}^{*}=O(R)$ and has to be computed numerically. Thus if $\operatorname{Pr}=0.72, a_{2}^{*} \sim-0.0067 R$ as $R \rightarrow x$ the 
mean flow distortion making a contribution $\sim-0.300 R^{2 / 3}$. More generally $a_{2}^{*}$ changes sign as $\operatorname{Pr}$ passes trough unity. It may also be shown that $a_{4}^{*}$ $=O\left(R^{2}\right)$ and when $\operatorname{Pr} \neq 1$ must be neglected in (5.33). It follows that the basic flow is supercritically stable if $\operatorname{Pr}<1$ and supercritically unstable if $\operatorname{Pr} \rightarrow 1$. Further. $:=O\left[\left(\frac{1}{4}-R i\right)^{1 / 2} R^{-1 / 2}\right\rceil$. the time-scale for the evolution of $A^{*}$ is $O\left[\left(\frac{1}{2}-R i\right)^{-1}\right]$ and the conditions for the validity of the theory are

$$
1 \ll\left(\frac{1}{4}-R i\right) R \ll R^{2 / 3},
$$

on use of (5.31).

If $\operatorname{Pr}=1$ an analytic study is possible and shows that $a_{2}^{*}=O\left(R^{1 / 3}\right)$ at most while the computations indicate that $a_{2}^{*} \rightarrow 0$ as $R \rightarrow \infty$. The terms involving $a_{4}^{*}$ must now be restored to (5.33) so that the amplitude equation more resembles (5.28); the restrictions (5.34) on $R i$ and $R$ are, however, unchanged.

5.4. Critical layer absorption. Let us now turn to the changes induced by a stable strongly-stratified shear layer on an incident wave. This question was first considered by Booker and Bretherton (1967) who noted that according to linear theory the incident wave does not penetrate beyond the critical layer and that the reflected and transmitted waves are exponentially small. They referred to this phenomenon as critical layer absorption and we have already briefly referred to the role played by the continuous spectrum in this mechanism.

In a series of papers, Brown and Stewartson (1980, 1981a,b) have carried out a detailed analysis of the process taking into account both linear and non-linear terms. In the first of these studies the analysis is closely similar to that described in Section 5.4. It is first shown that the incident wave $\psi_{1}$ may be represented near the critical level, which may also be taken as $y=0$ without any loss, by an expression similar to (5.15) namely

$$
\psi_{1}=A e^{i x x} \int_{0}^{a t} \frac{e^{i v u}}{u^{3 / 2}-i v} d u+B e^{i \alpha x} \int_{0}^{*} \frac{e^{-i y u}}{u^{3 / 2-i v}} d u+\text { c.c. }
$$

where $v=\left(J_{c}-\frac{1}{4}\right)^{1 / 2}, J_{c}$ is the Reynolds number at this level, $2 \pi / \alpha$ is the wavelength in the $x$-direction of the incoming wave and $A, B$ are constants determined from the properties of this wave. For $J_{c} \gg 1, B=O\left(A e^{-2 v \pi}\right)$ and may be neglected. The reflected and transmitted waves have similar forms but the corresponding values of $A$ and $B$ have different properties. It is clear from (5.35) that $\lambda \psi_{1} / \hat{\gamma} y \sim t^{1 / 2}$ as $t \rightarrow x$ so eventually non-linear terms must become important near $y=0$. These may be found by repeated substitution of (5.35), together with an equivalent form for $T$, into (5.1). Formally we obtain $\psi$ and $T$ as a series in powers of $\varepsilon t^{3 / 2}$ whose coefficients are functions of $x$ and $y t$, where $\varepsilon$ is the amplitude of the incident wave, but explicit 
expressions for them rapidly become too complicated to compute. It is easier to determine expressions for the reflection coefficient $\mathscr{A}$ and transmission coefficient $\mathscr{T}$ explicitly to order $\varepsilon^{2} t^{3}$ and we have, when $U(y)=\tanh y$, $R^{\prime}(y)=-1$,

$$
\begin{aligned}
& h=-i 2^{1 / 2-2 i v} e^{-v \pi}- \\
& \quad-\varepsilon^{2} 2^{i v-7 / 2} 5^{-1 / 4}(1+\sqrt{5})^{-5 i v-7 / 2} e^{2 i v} v^{-1-2 i v} t^{3+2 i v}
\end{aligned}
$$

while. $\bar{T}$ is exponentially small.

In the two later papers Brown and Stewartson exploit the simplifications which may be made directly in (5.35) when $v \gg 1$. By means of steepest descent arguments we may show that

$$
\begin{gathered}
\int_{0}^{a t} \frac{e^{-i y u}}{u^{3 / 2-i v}} d u \sim\left(\frac{2 \pi}{v}\right)^{1 / 2}\left(\frac{y}{v}\right)^{1 / 2-i v} e^{-i v-i \pi / 4}+ \\
+\frac{i e^{-i v \eta}(\alpha t)^{i v-1 / 2}}{v(\eta-1)}, \text { if } \eta>1, \\
\quad \int_{0}^{\alpha t} \frac{e^{-i y u}}{u^{3 / 2-i v}} d u-\frac{i e^{-i v \eta}(\alpha t)^{i v-1 / 2}}{v(1-\eta)}, \text { if } \eta<1,
\end{gathered}
$$

where $\eta=\alpha y t / v$. Of the terms on the right-hand side of $(5.37 \mathrm{a})$ the term independent of $t$ represents an incoming wave while the others may be identified with the continuous spectrum (5.11a), when $\eta \gg 1$, i.e., outside the neighbourhood of the critical layer. Thus, while in general the continuous spectrum may be identified with the genesis of the disturbance and may be neglected at large times, this is not the case near the critical layer. Here the contribution from the continuous spectrum, which may be called critical-level noise (CL-noise), is as important as the incident wave at all times and indeed the line $\eta=1$ acts as a transition point converting the incident wave into CL-noise. The form of (5.37) also enables us to give a physical interpretation of critical level absorption. It is the conversion of a wave carrying energy across the layer into CL-noise in which the energy is carried along the layer. This property may be seen more clearly by noting that the appropriate form of $\psi$ is then given by (5.11a). Consequently if the incident wave is arbitrary the appropriate form of $\psi$ is

$$
\psi \sim t^{-3 / 2} \cos \left(v(y) \log t+h_{1}(y)\right) h_{2}(y) f(x-t U(y))
$$

where $h_{1}, h_{2}, f$ are arbitrary functions. Strictly (5.37) give the leading terms of an asymptotic expansion in descending powers of $v$ all terms being similar in form to those given. There are, however, also terms of order $e^{-v \pi}$ which are systematically neglected but make a different contribution to the form of $\psi$. 
Using expressions like (5.37) Brown and Stewartson were able to carry out the expansion of the solution of $\psi$ to a much higher order in powers of $t$ than with their previous broad-brush method. For details reference should be nade to the orginal papers but we can report their conclusions. The reflected and transmitted waves may be written as a double series in powers of $\varepsilon t^{3 / 2}$ and $e^{ \pm i z x}$ whose coefficients are function of $y, v$ and integral powers of $t^{ \pm i v}$. his structure is similar to that arising when an incident wave strikes an unstratified shear layer but which has planetary vorticity, discussed in section 3. Outside the shear layer the form $\psi_{R}$ taken by the reflected wave is

$$
\psi_{R}=\sum_{n=0}^{N} \sum_{m=0}^{\infty} c_{n m}(v)\left(\varepsilon^{2 / 3} \alpha t\right)^{3(n+2 m+1) / 2} \cos \left\{n \alpha x+d_{n m}(v t)+k_{n} y\right\}
$$

:vhere $c_{n m}$ are algebraic functions of $v$ with leading terms $\sim 0.065^{-1}$ for $\because 10$ and $\sim 0.009^{-3 / 2}$ for $c_{20} ; d_{n m}$ are algebraic functions of $v, \log v$ and iog $t ; k_{n}=+\left(-\operatorname{RiR}(\infty)-n^{2} \alpha^{2}\right)^{1 / 2} ; N$ is the maximum value of $n$ for which SiR $(\infty)+n^{2} \alpha^{2}<0$.

The transmitted wave takes the form $\psi_{T}$ outside the shear layer where

$$
\psi_{T}=f_{11}\left(\varepsilon^{2 / 3} \alpha t\right)^{12} \cos \left(\alpha x+k_{1} y+g_{11}\right)+\ldots
$$

and $f_{11}, q_{11}$ are functions of $v$ only.

5.6. Discussion. The results we have presented about non-linear critizal layers do not exhaust the advances made in this area of stability theory. iar from it, for the problems raised are of considerable importance in ineteorology and oceanography and have therefore been the subject of intense study over recent years. They are believed to be associated with some of the thin layers of turbulence observed in the atmosphere and the ocean ihermocline regions (Browning et al. (1973); Thorpe and Hall (1974)), with lear air turbulence (Maslowe (1972)), and with the occurence of quasisermanent features of Jupiter's atmosphere such as the Great Red Spot Redekopp (1977)). Many of these studies, however, while throwing general light on various natural phenomena, have drawbacks when considered in the :ontext of critical-layer dynamics: Thus numerical studies must be carried sut in sufficient detail to give the structure of the critical layer and yet the Reynolds number must be large enough so that in some sense the flow may :e regarded as almost inviscid, an essential requirement to the main thrust of this article. The extra complication introduced with stratification does not make it easy to develop numerical schemes with the penetrating power of those used by Warn and Warn (1978) and Béland (1979). One of the most interesting is due to Patnaik, Sherman and Corcos (1976) who examined the evolution of a small disturbance to a stratified shear flow in a marginally unstable state. Considerable detail of the structure of the critical layer was noted including the formation of cat's eyes and the generation of "braids", 
expressions for them rapidly become too complicated to compute. It is easier to determine expressions for the reflection coefficient $\mathscr{A}$ and transmission coefficient $\mathscr{T}$ explicitly to order $\varepsilon^{2} t^{3}$ and we have, when $U(y)=\tanh y$, $R^{\prime}(y)=-1$,

$$
\begin{aligned}
& \not h=-i 2^{1 / 2-2 i v} e^{-v \pi}- \\
& -\varepsilon^{2} 2^{i v-7 / 2} 5^{-1 / 4}(1+\sqrt{5})^{-5 i v-7 / 2} e^{2 i v} v^{-1-2 i v} t^{3+2 i v}
\end{aligned}
$$

while $\mathscr{T}$ is exponentially small.

In the two later papers Brown and Stewartson exploit the simplifications which may be made directly in (5.35) when $v \gg 1$. By means of steepest. descent arguments we may show that

$$
\begin{gathered}
\int_{0}^{a t} \frac{e^{-i y u}}{u^{3 / 2-i v}} d u \sim\left(\frac{2 \pi}{v}\right)^{1 / 2}\left(\frac{y}{v}\right)^{1 / 2-i v} e^{-i v-i \pi / 4}+ \\
+\frac{i e^{-i v \eta}(\alpha t)^{i v-1 / 2}}{v(\eta-1)}, \text { if } \eta>1, \\
\int_{0}^{\alpha t} \frac{e^{-i y u}}{u^{3 / 2}-i v} d u--\frac{i e^{-i v \eta}(\alpha t)^{i v-1 / 2}}{v(1-\eta)}, \quad \text { if } \eta<1,
\end{gathered}
$$

where $\eta=\alpha y t / v$. Of the terms on the right-hand side of $(5.37 \mathrm{a})$ the tern. independent of $t$ represents an incoming wave while the others may $b$. identified with the continuous spectrum (5.11a), when $\eta \gg 1$, i.e., outside th neighbourhood of the critical layer. Thus, while in general the continuou: spectrum may be identified with the genesis of the disturbance and may $b$ : neglected at large times, this is not the case near the critical layer. Here th" contribution from the continuous spectrum, which may be called critical-leve; noise (CL-noise), is as important as the incident wave at all times anc: indeed the line $\eta=1$ acts as a transition point converting the incident wave: into CL-noise. The form of (5.37) also enables us to give a physica: interpretation of critical level absorption. It is the conversion of a wave carrying energy across the layer into CL-noise in which the energy is carried along the layer. This property may be seen more clearly by noting that the appropriate form of $\psi$ is then given by (5.11a). Consequently if the incident wave is arbitrary the appropriate form of $\psi$ is

$$
\psi \sim t^{-3 / 2} \cos \left(v(y) \log t+h_{1}(y)\right) h_{2}(y) f(x-t U(y))
$$

where $h_{1}, h_{2}, f$ are arbitrary functions. Strictly (5.37) give the leading terms of an asymptotic expansion in descending powers of $v$ all terms being similar in form to those given. There are, however, also terms of order $e^{-v \pi}$ which are systematically neglected but make a different contribution to the form of $\psi$. 
Using expressions like (5.37) Brown and Stewartson were able to carry out the expansion of the solution of $\psi$ to a much higher order in powers of $t$ than with their previous broad-brush method. For details reference should be made to the orginal papers but we can report their conclusions. The reflected and transmitted waves may be written as a double series in powers of $\varepsilon t^{3 / 2}$ and $e^{ \pm i \alpha x}$ whose coefficients are function of $y, v$ and integral powers of $t^{ \pm i v}$. This structure is similar to that arising when an incident wave strikes an unstratified shear layer but which has planetary vorticity, discussed in Section 3. Outside the shear layer the form $\psi_{R}$ taken by the reflected wave is

$$
\psi_{R}=\sum_{n=0}^{N} \sum_{m=0}^{\infty} c_{n m}(v)\left(\varepsilon^{2 / 3} \alpha t\right)^{3(n+2 m+1) / 2} \cos \left\{n \alpha x+d_{n m}(v t)+k_{n} y\right\}
$$

where $c_{n m}$ are algebraic functions of $v$ with leading terms $-0.065^{-1}$ for $c_{10}$ and $\sim 0.009^{-3 / 2}$ for $c_{20} ; d_{n m}$ are algebraic functions of $v, \log v$ and $\log t ; k_{n}=+\left(-R i R(\infty)-n^{2} \alpha^{2}\right)^{1 / 2} ; N$ is the maximum value of $n$ for which $\operatorname{RiR}(\infty)+n^{2} \alpha^{2}<0$.

The transmitted wave takes the form $\psi_{T}$ outside the shear layer where

$$
\psi_{T}=f_{11}\left(\varepsilon^{2 / 3} \alpha t\right)^{12} \cos \left(\alpha x+k_{1} y+g_{11}\right)+\ldots
$$

and $f_{11}, q_{11}$ are functions of $v$ only.

5.6. Discussion. The results we have presented about non-linear critical layers do not exhaust the advances made in this area of stability theory. Far from it, for the problems raised are of considerable importance in meteorology and oceanography and have therefore been the subject of intense study over recent years. They are believed to be associated with some of the thin layers of turbulence observed in the atmosphere and the ocean thermocline regions (Browning et al. (1973); Thorpe and Hall (1974)), with clear air turbulence (Maslowe (1972)), and with the occurence of quasipermanent features of Jupiter's atmosphere such as the Great Red Spot (Redekopp (1977)). Many of these studies, however, while throwing general light on various natural phenomena, have drawbacks when considered in the context of critical-layer dynamics: Thus numerical studies must be carried out in sufficient detail to give the structure of the critical layer and yet the Reynolds number must be large enough so that in some sense the flow may be regarded as almost inviscid, an essential requirement to the main thrust of this article. The extra complication introduced with stratification does not make it easy to develop numerical schemes with the penetrating power of those used by Warn and Warn (1978) and Béland (1979). One of the most interesting is due to Patnaik, Sherman and Corcos (1976) who examined the evolution of a small disturbance to a stratified shear flow in a marginally unstable state. Considerable detail of the structure of the critical layer was noted including the formation of cat's eyes and the generation of "braids", 
i.e., rapid density variations over half their boundaries. The Reynolds numbers chosen for the calculations were fairly small $(R \sim 100)$ and as a result both the disturbances and the basic flow decay until, at the end of the calculations, the density in the cat's eyes is almost constant.

Another interesting set of calculations has been carried out by Fritts $(1978,1979)$ at a much higher Reynolds number $\left(\sim 10^{3}\right)$ which reveals a number of intriguing aspects of the interplay between non-linearity and viscosity in stable flow fields $(R i>1)$. Unfortunately, from our point of view, he chooses a step length across the shear layer which is of the same order of magnitude as the width of the critical layer viewed either as inviscid or viscous. It is, of course, an open question as to whether this step length is adequate for the understanding of the large-scale phenomena in the shear layer, but it cannot give the fine structure which we know from the extensive studies reported here is characteristic of critical layers at high Reynolds number and small disturbances to the basic flow.

On the analytic side, two papers have been written (Kelly and Maslowe (1970); Maslowe (1972)) attempting to adapt the Benney and Bergeron theory to stratified flows so as to describe free oscillations when viscous effects are weak but persistent in the critical layer. However, these studies are incomplete because the importance of enforcing the Haberman option (1972) was not realized at that time. Subsequently, Haberman (1973) discussed the modifications to the mean shear produced by the oscillations but further work needs to be done to assess its significance. This will be well worthwhile, since it may be possible to set up free linear oscillations in the shear flow and outside the critical layer even when $R i>\frac{1}{4}$, the matching across the critical layer being effected by non-linear means, and perhaps being equivalent to a zero velocity jump. More recently, Huerre (1978) (see also Huerre and Scott (1980)). studied critical layers using Hadamard's concept of the finite part of a infinite integral and obtained an equation equivalent to (5.30) for the non-linear growth of waves which are neutral but weak. However, the basic state is unstable so that consideration needs to be given to the question whether their non-linear growth would be swamped by that of other waves.

Acknowledgment. It is a pleasure for me to thank Dr S. N. Brown for her very considerable help at all stages of the preparation of this survey.

\section{References}

[1] D. J. Acheson (1973), Valve effect of inhomogeneities on anistropic wave propagations, J. Fluid Mech. 58, 27--37.

[2] W. H. H. Banks, P. G. Drazin, and M. B. Zaturska (1976), On the normal modes of parallel fow of inviscid strarified fluid, J. Fluid Mech. 75, 149-171.

[3] M. Béland (1976), Numerical study of the non-linear Rossby wave critical level development in a barotropic zonal How, J. Atmospheric Sci. 33, 2066- 2078. 
[4] - (1978), The evolution of a non-linear Rossby wave critical layer: effects of viscosity, J. Atmospheric Sci. 35, 1802-1815.

[5] D. Benney and R. E. Bergeron (1969), A new class of non-linear waves in parallel flows, Stud. Appl. Math. 48, 181-204.

[6] J. R. B ooker and F. P. Bret herton (1967), The critical layer for internal gravity waves in a shear flow, J. Fluid Mech. 27, 513-539.

[7] S. N. Brown and K. Stewartson (1978), The evolution of the critical layer of a Rosshy wave, II, Geophys. Astrophys. Fluid Dynamics 10, 1-24.

[8] -, - (1979), On the secular stability of a regular Rossby neutral mode, Geophys. Astrophys. Fluid Dynamics 14, 1-18.

[9] -, - (1980), On the algehraic decay of disturhances in a stratilied linear shear How, J. Fluid Mech. 100, 811-816.

[10] -, - (1980). On the non-linear reflection of a gravity wate at a critical level, I, J. Fluid Mech. 100, $577-595$.

[11] -, - (1982), On the non-linear reftection of a gravity wave as a critical level, $I I$, Fluid Mech. 115, 217-230.

[12] -, - (1982), On the non-linear reflection of a gravity wate at a critical level. III, Fluid Mech. 115. 231-250.

[13] S. N. Brown, A. S. Rosen and S. A. Maslowe (1981), The erolution of a quasi steady. critical layer in a stratified viscous shear layer, Proc. Roy. Soc. London Ser. A., 375, 271293.

[14] K. A. Browning, G. W. Bryant, J. R. Starr, and D. N. Axford (1973), Air motion within Kelvin-Helmholtz billows determined from simultaneous Doppler radar and aircraft measurements, Quart. J. Roy. Met. Soc. 99, 608-618.

[15] K. M. Case (1961), Hydrodynamic stability and the inviscid limit, J. Fluid Mech. 10, 420 429.

[16] G. Chimonas (1979), Algebraic disturbances in stratified shear flows, J. Fluid Mech. 90. 1 19.

[17] A. Davey (1973), A simple numerical method for solving Orr Sommerfeld problemi, Quart. J. Mech. Appl. Math. 26, 401-411.

[18] - (1978), Private communication.

[19] R. E. Davis (1969), On the high Reynolds number How over a wavy houndary, J. Fluid Mech. 36, 337-346.

[20] R. E. Dickinson (1970), Development of a Rosshy wate critical level, J. Almospheric Sci. 27, 627-633.

[21] P. G. Drazin and L. N. Howard (1966), Hydrodynamic stability of parallel flow of inviscid Huid, Adv. in Appl. Mech. 9, 1-89.

[22] A. Eliassen, E. Höiland and R. Riis (1953), Two-dimensional perturbarion of a flow with constant shear of a stratified fluid. Institute for Weather and Climate Research, The Norwegian Academy of Sciences and Letters Pub. No. 1

[23] J. R. Foot e and C. C. Lin (1950), Some recent investigations in the theory of hydrodynamic ssability, Quart. Appl. Math. 8, 265- 280.

[24] D. C. Fritts (1978), The non-linear gravity wave critical level interaction, J. Atmospheric Sci. 35, 397-413.

[25] - (1979), The excitation of radiating waves and Kelvin-Helmholtz instabilities by the gravity wave-critical level interaction, J. Almospheric Sci 36, 12-23.

[26] D. C. Fritts and M. A. Geller (1976), Viscous stabilisation of gravity wave critical layers, J. Atmospheric Sci. 33, 2276-2284.

[27] A. E. Gill and A. Davey (1969), Instabilities of a buoyancy-driven system, J. Fluid Mech. 35, 775- 798 .

[28] S. Goldstein (1931), On the stability of superposed streams of different densities, Proc. Roy. Soc. London Ser. A 132 524-548. 
[29] P. Groen (1948), Contribution to the theory of internal waves, Kon. Ned. Met. Inst. Mededlingen Verhandelingen $B 125$.

[30] C. E. Grosch and H. Salwen (1968), The stability of steady and time-dependent plane Poiseuille flow, J. Fluid Mech. 34, 177-205.

[31] R. Haberman (1972), Critical layers in parallel flows, Stud. Appl. Math. 51, 139-161.

[32] - (1973), Wave induced distortions of a slightly stratified shear flow: a nonlinear criticallaver effect. J. Fluid Mech. 58, 727-736.

[33] - (1976), Non-linear perturbations of the Orr-Sommerfeld equation-asymptotic expansion of the logarir/mic phase shift across the critical laver. SIAM J. Math. Anal. 7, 70-81.

[34] N. Haurwitz (1931), Zur Theorie der Wellenhewegungen in Luft und Wasser, Veroffentl. Geophys. Inst. Univ. Leipzig 6.

[35] P. Hazel (1972), Numerical studies of the stability of inviscid stratified shear flows, J. Fluid Mech. 51, 39-61.

[36] L. N. Howard (1961), Note on a paper by J. W. Miles, J. Fluid Mech. 10, 509-512.

[37] - (1963). Neutral curces and stability boundaries in stratiffed flow, J. Fluid Mech. 16, 333342.

[38] - (1964), The number of instability modes in hydrodynamic stability problems, J. Mécanique 3, 433-443.

[39] L. N. Howard and P. G. Drazin (1964), On the stability of parallel flow of inviscid fluid in a rolating system wilh tariable Coriolis parameter, J. Math. Phys. 43, 83-99.

[40] P. Huerre (1978), The non-linear stability of a free shear layer in the viscous critical layer regime, Philos. Trans. Roy. Soc. London Ser. A 293, 643-675.

[41] P. Huerre and J. F. Scott (1980), Effects of critical layer structure on the non-linear evolution of waves in free shear layers. Proc. Roy, Soc. London Ser. A 371, 509-524.

[42] R. E. Kelly and S. A. Maslowe (1970), The non-linear critical layer in a slightly stratified shear How, Stud. Appl. Math. 49, 301-326.

[43] W. T. Lakin, B. S. Ng and W. H. Reid (1978), Approximations to the eigenvalue relation for the Orr-Sommerfeld prohlem, Philos. Trans. Roy. Soc. London Ser. A 289, 347-371.

[44] M. T. Landahl (1980), A note on an algebraic instability of inviscid parallel shear flows, J. Fluid Mech. 98, 243-251.

[45] M. Lessen, P. J. Singh and F. Paillet (1974), The stability of a trailing line vortex, Part I. Inviscid theory, J. Fluid Mech. 63, 753-763.

[46] D. K. Lilly (1966), On the instability of Ekman boundary flow, J. Atmospheric Sci. 23, 481494.

[47] C. C. Lin (1945), On the stability of iwo-dimensional parallel flows II, Stability in an inviscid Huid, Quart. Appl. Math. 3, 218-234.

[48] - (1955). The Theory of Hydrodynamic Stahility, Cambridge University Press.

[49] A. D. McEwan, D. W. Mander and R. K. S mith (1972), Forced resonant second order interaction between damped internal waves, J. Fluid Mech. 55, 589-608.

[50] L. M. Mack (1976), A numerical study of the temporal eigenvalue spectrum of the Blasius boundary layer, J. Fluid Mech. 73, 497-520.

[51] J. W. McK ay (1979), Private communication.

[52] S. A. Maslowe (1972), The generation of clear air turbulence by non-linear waves, Stud. Appl. Math. 51, 1-16.

[53] - (1974), Instability of rapidly rotating flows to non-axisymmetric disturbances, J. Fluid Mech. 64, 307-317.

[54] - (1977), Weakly non-linear stability theory of stratified shear flows, Quart. J. Roy. Met. Soc. 103, 769-783.

[55] J. M. Mihaljan (1962), A rigorous exposition of the Boussinesq approximation applicable 10 a thin layer of fuid, Astrophys. J. 136, 1126-1133.

[56] J. W. Miles (1961), On the stability of heterogeneous shear flows, J. Fluid Mech. 10, 496-508. 
[57] - (1963), On the stability of heterogeneous shear flows, Part II, J. Fluid Mech. 16, 209-227.

[58] J. W. Murdock and K. Stewartson (1977), Spectra of the Orr-Sommerfeld equation, Physics Fluids 20, 1404-1411.

[59] T. O'Neil (1965), Collisionless damping of non-linear plasma oscillations, Phys. Fluids 8, 2255-2262.

[60] P. C. Patnaik, F. S. Sherman and G. M. Corcos (1976), A numerical simulation of Kelvin-Helmholtz waves of finite amplitude, J. Fluid Mech. 73, 215-240.

[61] T. J. Pedley (1968), Instability of rapidly rotating shear flows to non-axisymmetric disturbances, J. Fluid Mech. 31, 603-607.

[62] J. Pedlosky and H. P. Greenspan (1967), A simple laboratory model for the oceanic circularion, J. Fluid Mech. 27, 291-304.

[63] L. G. Redekopp (1977), On the theory of solitary Rossby waves, J. Fluid Mech. 82 , 725.745.

[64] P. H. Roberts and K. Stewartson (1974), On finite amplitude convection in a rotating magnetic system, Philos. Trans. Roy. Soc. London Ser. A 277, 287-315.

[65] A. S. Rosen (1978), Private communication.

[66] F. T. Smith (1980), Private communication.

[67] J. T. Stuart (1960), On the non-linear mechanics of wave disturbance in stable and unstable parallel flows, Part I. The basic behaviour in plane Poiseuille flow, J. Fluid Mech. 9, 353-370.

[68] K. Stewartson (1978), The evolution of the critical layer of a Rossby wave, Geophys. Astrophys. Fluid Dynamics 9, 185-200.

[69] T. Tatsumi and K. Goto (1969), The structure of damping disturbances in the stability of unbounded laminar flows, Proc. IUTAM Symp. Stability Cont. Systems Herrenalb, 368375.

[70] G. I. Taylor (1931), Effect of variation of density on the stability of the superposed stream of fluid, Proc. Roy. Soc. London Ser. A 132 499--523.

[71] J. A. Thorpe and A. J. Hall (1974), Evidence of Kelvin-Helmholtz billows in Loch Ness, Limn. Oceanogr. 19, 973-976.

[72] O. Tietjens (1925), Beiträge zur Entstehung der Turbulenz, Z. Angew. Math. Mech. 5, 200-217.

[73] W. Tollmien (1935), Ein allgemeines Kriterium der Instabilität Laminaren Geschwindigkeitsverteilungen, Nachr. Geschwind. Wiss. Göttingen 50, 79-114.

[74] T. Warn and H. Warn (1976), Development of a Rossby wave critical level, J. Atmospheric Sci. 33, 2021-2024.

[75] - , - (1978), The evolution of a nonlinear critical level, Stud. Appl. Math. 59, 37-71.

[76] W. Wasow (1948), The complex asymptotic theory of a fourth order differential equation of hydrodynamics, Ann. of Math. 39, 852-871.

Presented to the semester

Marhematical Models and Methods

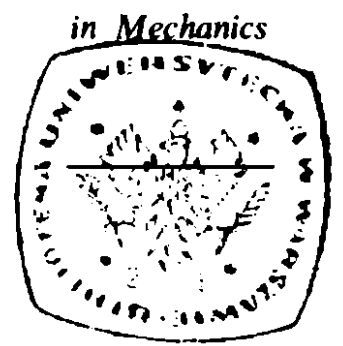

